\title{
Evolutionary signatures in complex ejecta and their driven shocks
}

\author{
C. J. Farrugia ${ }^{1}$ and D. B. Berdichevsky ${ }^{2}$ \\ ${ }^{1}$ University of New Hampshire, Space Science Center, Durham, NH, 03824, USA \\ ${ }^{2}$ L3 GSI, Government Service, Largo, MD, USA
}

Received: 11 March 2004 - Revised: 9 August 2004 - Accepted: 25 August 2004 - Published: 3 November 2004

\begin{abstract}
We examine interplanetary signatures of ejectaejecta interactions. To this end, two time intervals of innerheliospheric $(\leq 1 \mathrm{AU})$ observations separated by 2 solar cycles are chosen where ejecta/magnetic clouds are in the process of interacting to form complex ejecta. At the Sun, both intervals are characterized by many coronal mass ejections (CMEs) and flares. In each case, a complement of observations from various instruments on two spacecraft are examined in order to bring out the in-situ signatures of ejectaejecta interactions and their relation to solar observations. In the first interval (April 1979), data are shown from Helios2 and ISEE-3, separated by $\sim 0.33 \mathrm{AU}$ in radial distance and $28^{\circ}$ in heliographic longitude. In the second interval (MarchApril 2001), data from the SOHO and Wind probes are combined, relating effects at the Sun and their manifestations at $1 \mathrm{AU}$ on one of Wind's distant prograde orbits. At $\sim 0.67 \mathrm{AU}$, Helios-2 observes two individual ejecta which have merged by the time they are observed at 1 AU by ISEE-3. In March 2001, two distinct Halo CMEs (H-CMEs) are observed on SOHO on 28-29 March approaching each other with a relative speed of $500 \mathrm{~km} \mathrm{~s}^{-1}$ within 30 solar radii. In order to isolate signatures of ejecta-ejecta interactions, the two event intervals are compared with expectations for pristine (isolated) ejecta near the last solar minimum, extensive observations on which were given by Berdichevsky et al. (2002). The observations from these two event sequences are then intercompared. In both event sequences, coalescence/merging was accompanied by the following signatures: heating of the plasma, acceleration of the leading ejecta and deceleration of the trailing ejecta, compressed field and plasma in the leading ejecta, disappearance of shocks and the strengthening of shocks driven by the accelerated ejecta. A search for reconnection signatures at the interface between the two ejecta in the March 2001 event was inconclusive because the measured changes in the plasma velocity tangential to the interface $\left(\Delta \mathbf{v}_{t}\right)$ were not correlated with $\Delta\left(\mathbf{B}_{t} / \rho\right)$. This was possibly due to lack of sufficient magnetic shear across the interface. The ejecta mergers altered interplanetary parameters considerably, leading to contrasting geoeffects despite broadly similar solar activity. The complex ejecta on 31 March 2001 caused a double-dip ring current enhance-
\end{abstract}

Correspondence to: C. J. Farrugia

(charlie.farrugia@unh.edu) ment, resulting in two great storms $\left(D_{s t}\right.$, corrected for the effect of magnetopause currents, $<-450 \mathrm{nT}$ ), while the merger on 5 April 1979 produced only a corrected $D_{s t}$ of $\sim-100 \mathrm{nT}$, mainly due to effects of magnetopause currents.

Key words. Interplanetary physics (interplanetary magnetic fields; interplanetary shocks) - Magnetospheric physics (storms and substorms)

\section{Introduction}

A topic of increasing interest to heliospheric physics concerns what happens to the interplanetary (IP) counterparts of coronal mass ejections (ICMEs, called henceforth "ejecta", keeping the designation CME for the object as seen in coronagraphs) when they interact en route to Earth. Evidence that some do interact has been advanced recently in the form of an excitation of a broad-band type II radio burst emission observed by Wind/WAVES at the same time that the SOHO/LASCO coronagraph monitored a CME overtaking another one close to the Sun (Gopalswamy et al., 2001, 2002). Burlaga et al. (2002) considered configurations called "complex ejecta" which they showed to be due to CMEs released under such kinematic conditions that the ejecta collided with each other within $1 \mathrm{AU}$. One surprising aspect of these IP observations of complex ejecta at $1 \mathrm{AU}$ is their simple bulk speed profile, which essentially just declines steadily over a period of several days. This deceptively simple speed profile, however, masks an intricate internal structure where individual CMEs have merged and lost their separate identity. (Other examples of complex ejecta, composed this time of multiple magnetic clouds (Burlaga et al., 1981, 1990) were given by Wang et al. (2003).) The merger of ejecta alters the parameter profiles which IP probes measure and which eventually affect the Earth, and with it the geoeffectiveness of these large structures. In contrast to the duration of Earth passage of pristine ejecta (or "isolated" ejecta, in the sense of interacting solely with the background solar wind), which is typically of the order of 1 day (Klein and Burlaga, 1982; Gosling, 1990; Berdichevsky et al., 2002), the passage of complex ejecta may last up to $\sim 4$ days, so that the magnetosphere is immersed in unusual IP conditions for a much longer time. 
Simulations on how ejecta interact with the ambient solar wind have been carried out (Pizzo, 1997; Odstrcil and Pizzo, 1999a, b, c). However, little is known as to how the merger of ejecta develops in IP space and how the geoffective potential is altered. Is the geoeffectiveness of the whole less or more than that of the sum of its parts? This is the space weather issue involved.

This paper seeks to understand the evolutionary signatures involved when complex ejecta form. We do this by examining two complex ejecta separated almost exactly by two solar cycles, both observed close to the maximum phase of the solar activity cycle. Further, by employing in one event observation separated radially by $\sim 0.33 \mathrm{AU}$ on average, though offset from one another in longitude, we actually have a twopoint measurement of the evolutionary processes, allowing us to infer, in a snapshot fashion, the changes in parameters that take place.

For both time intervals similar levels of transient activity are present at the Sun. This activity includes more than $20 \mathrm{H} \alpha$ flares a day from regions within $40^{\circ}$ of central meridian, a comparable number of solar radio bursts, a similar level of background radiation in the 1-8 $\AA$ soft X-ray wavelength range, and the presence of long duration events (LDE). Both intervals include several CMEs, some of which were identified as Halo CMEs (H-CMEs). Notwithstanding this similar activity at the source, the two intervals gave rise to very disparate geoeffects at Earth, as we shall see.

Preliminary simulations on ejecta-ejecta interactions have been undertaken which we can compare against. Odstrcil et al. (2003) specialized in two magnetic clouds, whose fields when they come into contact are oppositely directed, which were overtaking each other. They found the interaction to involve acceleration (deceleration) of the leading (trailing) magnetic cloud, heating of the plasma, coalescence of the underlying two magnetic flux tubes into one flux tube by a reconnection process, and so forth (see the Summary and discussion section). Thus, we shall investigate if some of these effects are indeed present in our two event sequences. The paper shall conclude by emphasizing the importance of understanding these effects on a broad experimental basis as an essential component of the space weather program. Here we can present only snapshots, so that our interpretations are circumscribed by important caveats (see the Summary and discussion section).

A note on procedure follows. For pristine ejecta it was found (Berdichevsky et al., 2002) that at $1 \mathrm{AU}$ a) the ejecta drive the shocks, i.e. the shock speed is within the limits of error (i.e. within the Alfvén speed of the medium) approximately equal to the speed of the leading edge of the ejecta; and b) the ejecta as a whole retain their velocity while travelling from $\sim 2 R_{S}$ (solar radii) to $1 \mathrm{AU}$. Clear deviations from these findings, together with the presence of multiple ejecta in space, indicate departures from pristine conditions, i.e. interactions/collisions.

\section{Previous work}

We summarize here key results reached in other works on the events we study. The April 1979 events were investigated by Burlaga et al. (1987) in the context of compound streams, which are configurations produced by the interaction of two or more distinct fast flows, a concept introduced by Burlaga (1975). The components of the compound stream were identified at both Helios-2 and ISEE-3. The authors ascribed the configuration to ejecta containing a magnetic cloud overtaking ejecta not containing a magnetic cloud. Coalescence of ejecta material at ISEE-3 is inferred. A solar source was proposed for each of the components making up the compound stream.

Sun et al. (2002) modeled the travelling shocks during 28 March-21 April, using an MHD code (Haikamada-AkasofuFry model, Fry et al., 2001). The solar sources of the various ejecta in this period are identified, based on near-real time communication of SOHO/LASCO/EIT CMEs. The main aim of the paper was to use this code to predict the arrival of the shocks, as part of a space weather prediction effort. To bridge certain discrepancies between predictions and observations, they advocate the need for a 3-D coronal density model for applications to solar flares and their associated type II radio bursts, which is needed as input to their shock modeling. The authors conclude that shock speeds obtained from metric type 2 bursts may not have been accurate. Finally, they also link the flare times with sudden storm commencements at Earth.

A second paper which included a study of the MarchApril 2001 events was made by Wang et al. (2003). The authors define a new type of complex ejecta called "multiMCs" which consist of two or more ejecta, interpreted as magnetic clouds, and interaction regions between them. By visually inspecting the time profiles, they find that each component of a multi-cloud behaves like an isolated cloud with two exceptions: the temperature may be higher and the speed of the leading cloud at its trailing edge increases. They attribute these to ejecta-ejecta interaction. They also identify the CMEs responsible for the 2 ejecta seen at $1 \mathrm{AU}$ on 31 March. They also report that high $D_{s t}$ values were measured in two of the 3 case events studied.

Our analysis confirms these findings and adds new elements. These are: (i) a thorough quantitative analysis of the shocks. This is important since two major effects of the interaction relate to shocks: it strengthens the shock driven by the leading ejecta and weakens that originally driven by the trailing ejecta. (ii) We infer acceleration and deceleration using the concept of pristine ejecta and their propagation properties, thus following a different methodology from that of the previous works. Ours is an application of the work of Berdichevsky et al. (2002). (iii) Further to (ii), we also estimate the size of the average acceleration/decleration and from this obtain an estimate the relative masses of the ejecta. (iv) Using again the concept of pristine ejecta, we are able to distinguish those components of complex ejecta which were interacting from those which were not (i.e. are 
still pristine). (v) We compare observational results concerning signatures of interaction with predictions of numerical modeling on same. (vi) We isolate ejecta mergers as one interplanetary cause of double-dip storms with the property that the first storm is stronger than the second; (vii) Finally, our analysis is based on, to quote one referee,"perhaps the most comprehensive assimilation of the data sets".

\section{Observations in April 1979}

\subsection{Helios-2 observations}

Figure 1 shows an ecliptic projection of the Helios-1 (black trace) and Helios-2 orbits (blue trace) in 1979. The plot is drawn with a fixed Earth-Sun $(\mathrm{E}-\mathrm{S})$ line and is centered on the Sun. ISEE-3 (I3) is orbiting around the L1 Lagrangian point upstream of Earth $(R \sim 0.99 \mathrm{AU})$. The period under study is marked in red on the Helios traces. Helios- 2 is $\sim 28^{\circ}$ east of the Sun-Earth line and approaching the Sun, being at a radial distance of $0.69 \mathrm{AU}$ (2 April) and $0.66 \mathrm{AU}$ (end of 6 April). (The lines labeled S1-S3 are discussed below.)

We shall now examine the measurements at Helios-2 and ISEE-3. Figures 2 and 3 show in the same format (including the same range in the vertical scales) measurements of plasma, magnetic field, and energetic particles at Helios-2 and ISEE-3 made on 2-6 April 1979: proton density, bulk speed, and temperature, the (SE/GSE) components of the magnetic field and the total field strength, the dynamic pressure (based on the protons), the number density ratio $n_{\alpha} / n_{p}$ of $\alpha$-particles to protons in percent, the proton plasma beta, and the energetic $\mathrm{H}^{+}$flux in three channels as indicated. (The data in the ISEE-3 plot is from IMP-8, McGuire et al., 1986.) (In the solar ecliptic (SE) coordinate system the $\mathrm{x}$-axis points from the spacecraft to the Sun, the y-axis is positive east, and the $\mathrm{z}$-axis is perpendicular to the ecliptic such that (xyz) is a right-handed system.) The time scale in Fig. 3 is shifted forward by $12 \mathrm{~h}$ with respect to that in Fig. 2. ISEE-3 data are at 5-min temporal resolution, while Helios-2 are nominally at 4-s resolution, though the coverage is uneven. At Helios-1, displaced $42^{\circ}$ east of Helios-2, none of the ejecta are observed, setting thus an upper limit to their eastward extent. The red trace in the third panel is the expected temperature for normal solar wind expansion (after Lopez and Freeman, 1986; see also Steinitz and Menasche, 1982). In studies of pristine ejecta, temperatures substantially lower than this are considered to be a robust signature of ejecta material in space (Richardson et al., 1995, and references therein; see also Gosling et al., 1973).

Helios-2 sees a succession of four shocks, labeled S1-S4. Some hours after each shock passage, the following features are observed: strong fields, low proton beta, high and variable $n_{\alpha} / n_{p}$ ratio (except after shocks $\mathrm{S} 2$ and $\mathrm{S} 3$, where there are data gaps), and, in the case of the first ejecta, a magnetic cloud signature (i.e. a low-beta magnetoplasma in which a strong magnetic field rotates smoothly over a large angle, Burlaga et al., 1981). All these features indicate the presence

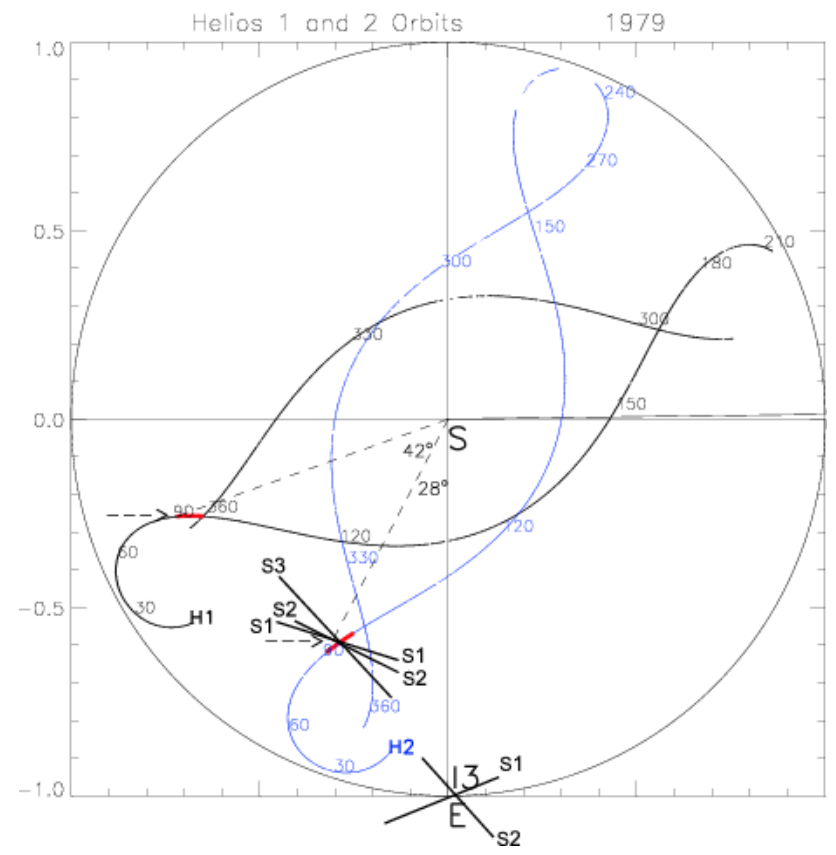

Fig. 1. The trajectories of Helios-1 (black trace) and Helios-2 during 1979, shown in a system with a fixed Sun-Earth line. The nearEarth spacecraft ISEE-3 is marked I3. The ecliptic projections of the shock fronts at both locations are also indicated.

of ejecta material behind each shock (see, e.g. Gosling, 1990, and references therein; Burlaga et al., 2001). We have labelled the ejecta E1-E4. The blue trace in panel 2 joins the midpoints of E1 and E3 and it is evident that there is a rising trend in velocity, which increases from $\sim 450 \mathrm{~km} \mathrm{~s}^{-1}$ to $\sim 750 \mathrm{~km} \mathrm{~s}^{-1}$ from 3 April to 5 April. The ejecta are in the process of overtaking each other (see Sect. 2.1 and Berdichevsky et al., 2003.) In particular, S3 is advancing into the rear of E2 and S4 is advancing into the rear of E3, as confirmed by the shock speeds computed below. Note that while the proton $\beta_{p}$ is low in each of the four ejecta, the proton temperature is not low in some of them. For example, it is not low in some regions of the ejecta E2 behind shock S2 (By "low" we mean "compared to the expected temperature"; red trace).

\subsection{Analysis of shocks S2 and S3 at Helios-2, and CME lift-off at the Sun}

There are many ways of computing shock normals and speeds. We use here the technique elaborated by Berdichevsky et al. (2000; see erratum, 2001), which combines the so-called "pre-averaged" magnetic coplanarity, velocity coplanarity, and the Abraham-Schrauner methods (Abraham-Schrauner, 1972). Basically, our method searches for a shock orientation where the Rankine-Hugoniot conditions are approximately satisfied. Among all possible solutions it requires further that there be agreement within $15^{\circ}$ with the shock normals from two of the pre-averaged methods. This technique has proved to be good when applied 


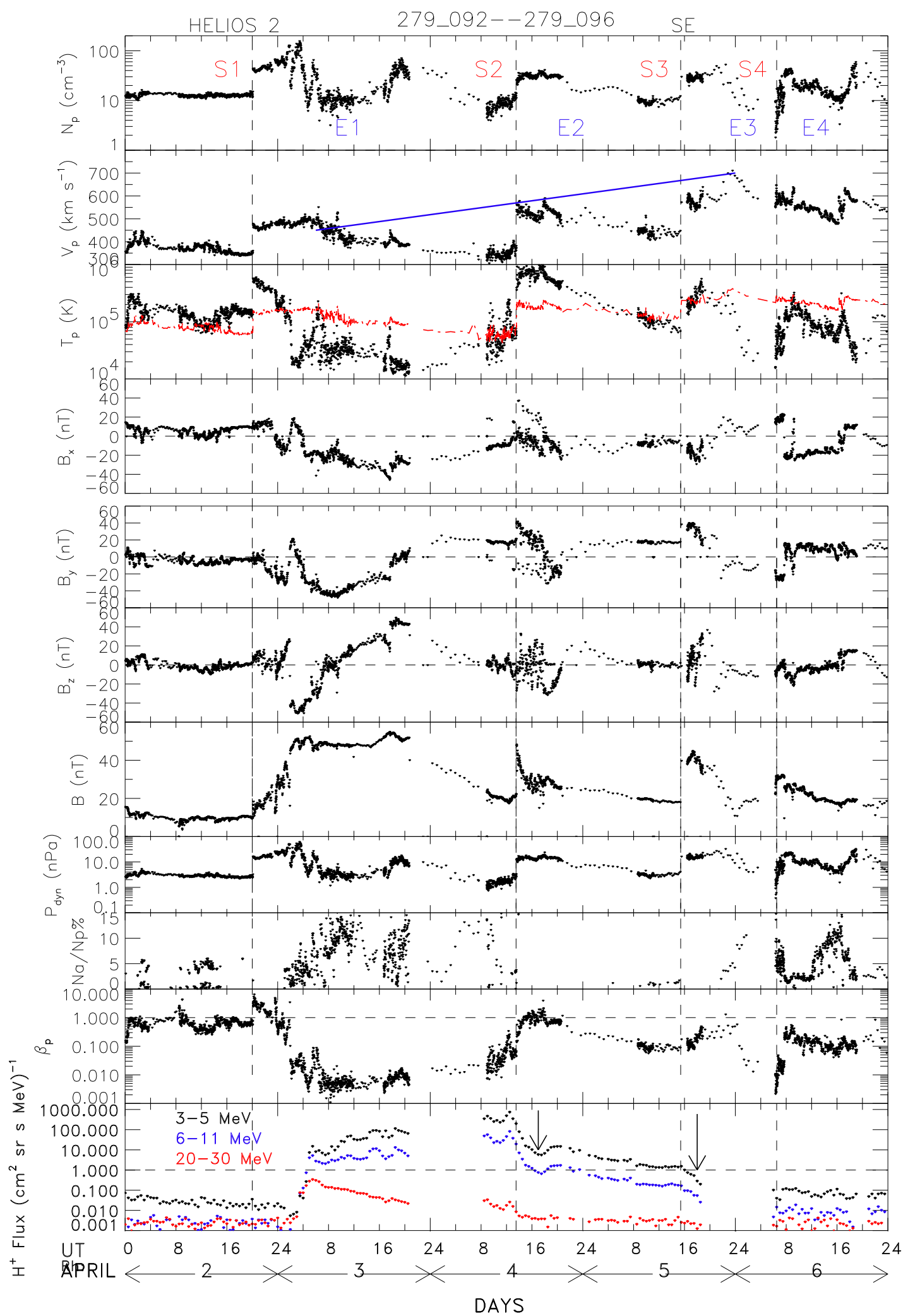

Fig. 2. Plasma, magnetic field, and energetic particle data for 2-6 April 1979. Shown from top to bottom are the proton density, bulk speed, and temperature, the components of the magnetic field in solar ecliptic (SE) coordinates, the total field strength, the dynamic pressure based on the protons, the $\alpha$-to-proton number density ratio in percent, the proton beta, and the proton fluxes in the MeV energy ranges shown. The red line in panel 3 gives the expected solar wind temperature for normal solar wind expansion. The blue line in panel 2 joins the estimated mid-point of the first and third ejecta. 


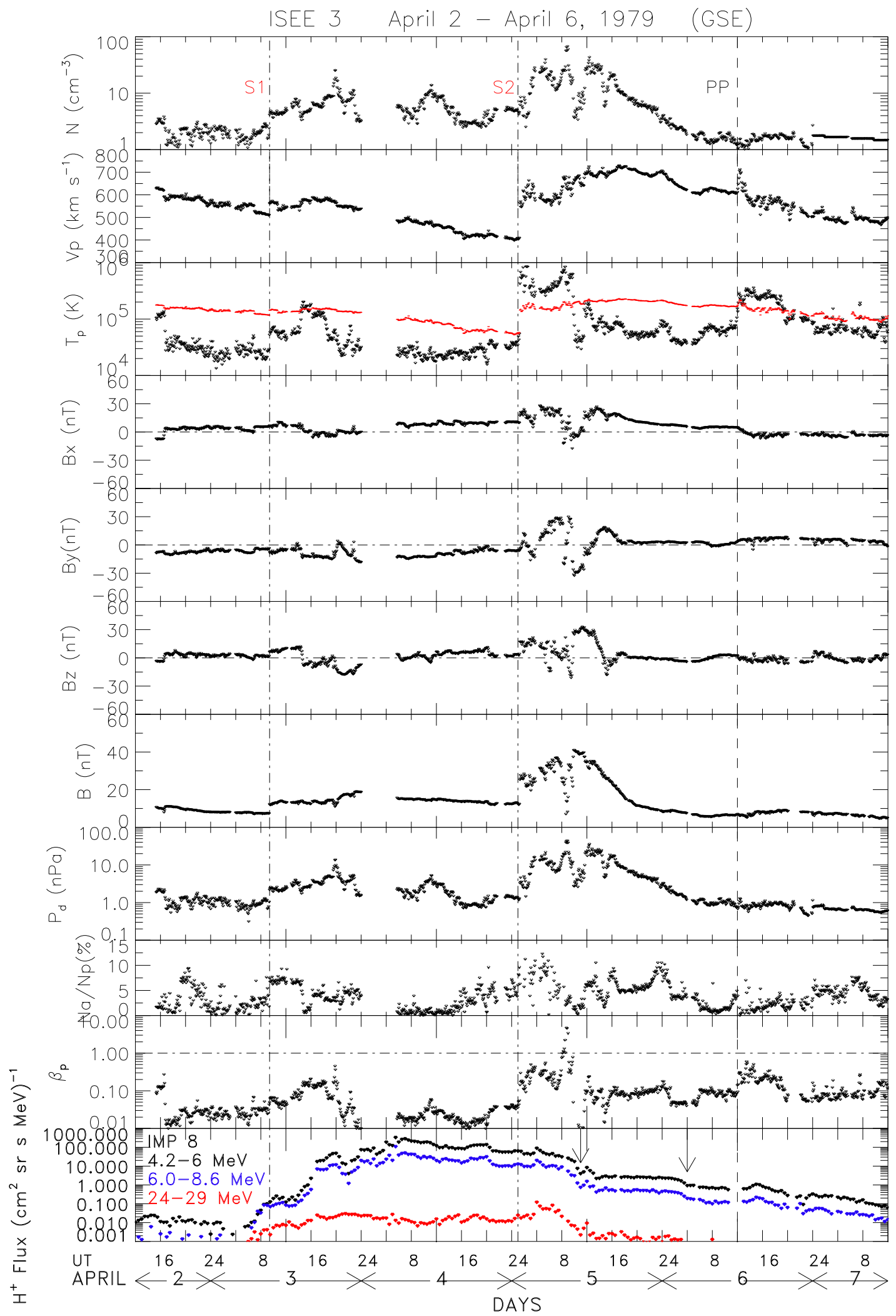

Fig. 3. Data acquired by ISEE-3 at $1 \mathrm{AU}$, presented in the same format as Fig. 2 . Note that the time axis is advanced by $12 \mathrm{~h}$ relative to that in Fig. 2. 
Table 1. Results of the analysis of the April 1979 shocks. Normal is the calculated shock normal. The next column shows the speed of each shock in $\mathrm{km} / \mathrm{s}$ with respect to the upstream solar wind $\left(V s^{\prime}\right)$ and in a solar inertial frame $(V s), \Theta_{B, n}$ is the angle between the upstream IMF vector and the shock normal; $B_{d w} / B_{u p}$ and $n_{d w} / n_{u p}$ are the compression ratios of the magnetic field strength and the density, respectively; $M s$ is the sonic Mach number, $\mathrm{R}$ and $\Phi$ are the heliospheric distance and longitude, respectively. All vector quantities are given in SE coordinates. During the passage of shock S3 at Helios-2 there are only a few data points so we do not derive an estimate of the uncertainty in the RH parameters (marked*).

\begin{tabular}{llcccccccccc}
\hline No. & $\begin{array}{l}\text { Day } \\
\text { April }\end{array}$ & $\begin{array}{l}\text { Time } \\
(\mathrm{UT})\end{array}$ & Normal & $\begin{array}{l}V s^{\prime} \\
\mathrm{km} \mathrm{s}^{-1}\end{array}$ & $\begin{array}{l}V s \\
\mathrm{~km} \mathrm{~s}^{-1}\end{array}$ & $\begin{array}{l}\Theta_{B, n} \\
\left({ }^{\circ}\right)\end{array}$ & $B_{d w} / B_{u p}$ & $n_{d w} / n_{u p}$ & $\begin{array}{l}M s \\
\text { SC } \\
(\mathrm{AU})\end{array}$ & $\begin{array}{l}\text { Loc. } \\
\left({ }^{\circ}\right)\end{array}$ \\
\hline Helios-2 & & & & & & & & & & & \\
1 & 2 & $20: 11$ & $(-0.97,-0.13,-0.24)$ & 176 & 523 & $9 \pm 2$ & $1.3 \pm 0.2$ & $2.8 \pm 0.2$ & 2.0 & 0.68 & $27 \mathrm{E} 7 \mathrm{~S}$ \\
2 & 4 & $13: 24$ & $(-0.98,0.0,0.15)$ & 273 & 640 & $83 \pm 5$ & $2.2 \pm 0.2$ & $2.5 \pm 0.4$ & 2.1 & 0.66 & $26 \mathrm{E} 7 \mathrm{~S}$ \\
3 & 5 & $15: 24$ & $(-0.94,0.35,0.0)$ & 262 & 690 & $48 \pm * *$ & $2.1 \pm * *$ & $2.5 \pm * *$ & 2.2 & 0.65 & $26 \mathrm{E} 7 \mathrm{~S}$ \\
ISEE-3 & & & & & & & & & & & \\
1 & 3 & $09: 22$ & $(-0.92,-0.38,-0.40)$ & 149 & 653 & $54 \pm 2$ & $1.7 \pm 0.2$ & $1 \pm 0.2$ & 1.6 & 0.99 & $0 \mathrm{E} 0 \mathrm{~S}$ \\
2 & 5 & $01: 12$ & $(-0.64,0.72,0.28)$ & 265 & 690 & $40 \pm 5$ & $2.0 \pm 0.2$ & $2.8 \pm 0.5$ & 2.1 & 0.99 & $0 \mathrm{E} 0 \mathrm{~S}$ \\
\hline
\end{tabular}

at shock passages with multiple spacecraft data. It has shown the same rate of failure as the post-averaged RankineHugoniot method of Vinas and Scudder (1986) and Szabo (1994). The error analysis used is a statistical one that combines a number of normal evaluations for different upstream and downstream intervals.

Shocks S2 and S3 play a very central role in our comparison and so we analyze them first. All results of this section are listed in Table 1, and the shock fronts are also shown in Fig. 1. Shock S2 passed Helios-2 at 13:24 UT (4), 1979. (Henceforth in this section we shall use for convenience the notation xx UT (y) to denote xx UT on April y). We obtain a shock normal $\mathbf{n}_{2}=(-0.98,0.0,0.15)$ (SE coordinates) and a shock speed of $640 \mathrm{~km} \mathrm{~s}^{-1}$ in a solar inertial frame. The shock compression ratio is moderate $(\sim 2.2$ in the magnetic strength, $B$, and plasma density, $n_{p}$ ), and the shock is quasi-perpendicular with $\theta_{\mathbf{B}, \mathbf{n}_{2}} \approx 83^{\circ}$, where $\mathbf{B}$ is the upstream interplanetary magnetic field (IMF). The shock orientation and speed suggest that locally the shock is propagating radially away from the Sun, consistent with its being driven by the leading edge of the ejecta E2, which passed Helios-2 at $\sim 18: 00$ UT (4) with a speed of $600 \mathrm{~km} \mathrm{~s}^{-1}$. At 04:00 UT (5) the midpoint of ejecta E2 passed Helios-2 with a speed of $\sim 530 \mathrm{~km} \mathrm{~s}^{-1}$. Assuming pristine conditions, this would place the lift-off time of E2 from the Sun at approximately 00:00 UT (3).

Around this inferred lift-off time there are the following solar signatures. From 01:05 to 03:30 UT (3), there is an $\mathrm{H} \alpha$ optical flare of importance $1 \mathrm{~B}$ at S25W13, associated with a soft X-ray M5, very long duration event, typically associated with large CMEs. Also, Culgoora, Australia reports from 01:30 UT to 05:30 UT a possible metric type IV radio burst, also suggestive of a CME. In the list complied by Cane (1985), a strong shock association is indicated with the start at 01:10 UT (3) of a metric type II radio burst (See Fig. 4). As the bottom panel of Fig. 2 shows, a moderate SEP event starts at about 03:00 UT (3), which appears to be associated with shock S2. Finally, the solar
Maximum Mission coronagraph lists (at URL http:www.hao. ucar.edu/public/research/svosa/smm/smmcp_catalog.html) a fast CME observation at 01:15 UT (3) with a plane-of-thesky speed of $1000 \mathrm{~km} \mathrm{~s}^{-1}$ toward the east. Thus, our estimate of lift-off time of ejecta E2, namely 00:00 UT (3), appears to be reasonable, supporting the "pristine" assumption.

Shock S3, which passed Helios-2 at 15:24 UT (5), 1979, has a shock normal $\mathbf{n}_{3}=(-0.94,0.35,0.0)$ in SE coordinates and a speed of $690 \mathrm{~km} \mathrm{~s}^{-1}$ in a solar inertial frame. The shock compression is again moderate $\left(\sim 2.1\right.$ in $B$ and $\sim 2.5$ in $\left.n_{p}\right)$, and the shock is orientated obliquely to the upstream IMF with $\theta_{\mathbf{B}, \mathbf{n}_{3}} \approx 48^{\circ}$. The measurements of the shock orientation and speed suggest that the shock has locally a somewhat eastward but mainly a radial direction of motion, consistent again with its being driven locally by the leading edge of the ejecta E3, which had a speed $\approx 700 \mathrm{~km} \mathrm{~s}^{-1}$ when it passed Helios-2 at $\sim 22: 30$ UT (5). The orientation of S3 is consistent with a solar source at the west of the Sun. If we were to assume E3 to be a pristine ejecta with the observed mid-point speed, we would place the lift-off of the CME at about 2 solar radii from the solar surface at approximately 10:00 UT (4).

Around this inferred time there are no clear signatures at the Sun in the vicinity of a central meridian. However, near 08:00 UT (4) there is a soft X-ray LDE C8 flare (see Fig. 4). The observed optical flare lasts from 07:20 to 07:40 UT with importance 1B at S22W56. It shows multiple bright points. This matches the predicted lift-off time reasonably well. The optical flare shows several brilliant points. It is associated with a system of loops which are prominence-like (Solar Geophys Data, 1980; Burlaga et al., 1987). No other comment is added. This event would be related to Helios-2 solar wind observations only if the flare location marks the western footpoint of the ejecta, and the eastern footpoint was not seen because of the ejecta being oriented toward the Earth's direction. Near 10:00 UT there is a minor LDE soft X-ray enhancement without a counterpart observation of an $\mathrm{H} \alpha$ flare. (This interval is not covered by the Solar Maximum Mission coronagraph. It is located in the middle of a 3-day gap in the 


\section{April 1979}

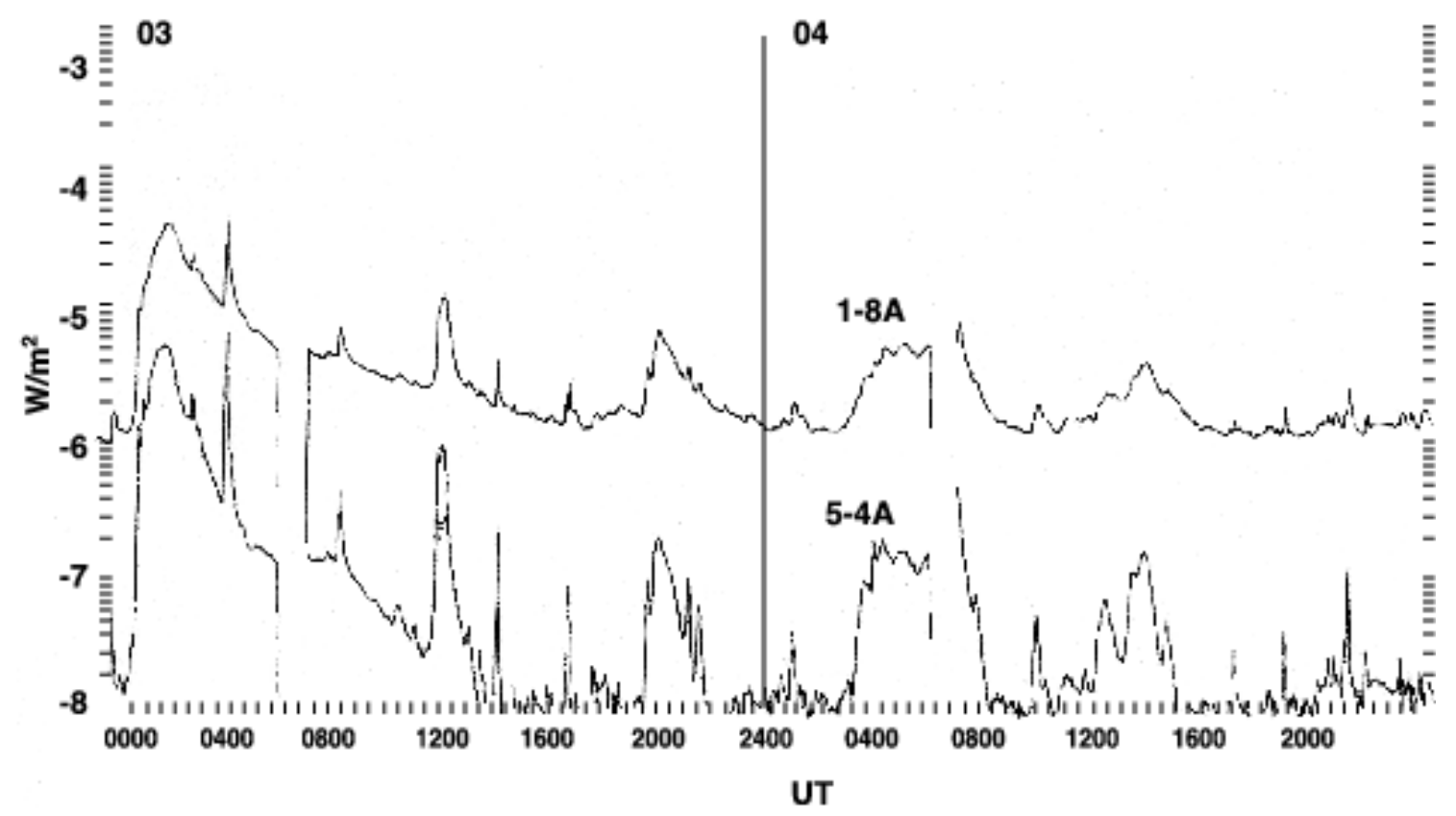

Fig. 4. SMS-GOES 8 measurements of soft X-rays in 2 wavelengths on 3-4 April 1979.

listing of observed CMEs. Nor is there in Cane's (1985) list a type II radio burst in association with 4 April 1979.)

\subsection{ISEE-3 observations}

Our two-site observations at large separation permit a later "snapshot" of this interaction of ejecta, for which we now consider the ISEE-3 measurements, shown in Fig. 3. Shock $\mathrm{S} 1$ is observed by ISEE-3 $13.5 \mathrm{~h}$ after it passed Helios-2. Shock $\mathrm{S} 2$ is also present, observed at the time indicated by the second vertical guideline. The time interval S1-S2 at ISEE-3 (40 h) is comparable to that at Helios-2 (41 h), which provides a good consistency check on these shock associations. Shock S4, which was weak at Helios-2, may have passed ISEE3 at 12:00 UT (6) (marked "PP"). We believe it is much weaker and in the form of a pressure pulse.

The declining bulk speed profile at ISEE-3 (panel 2), of similar duration as at Helios-2, has a double-peak feature, i.e. the configuration is now a compound stream (e.g. Burlaga, 1990). The impulsive rises in the solar wind $v$-profile due to the fact that various shocks have disappeared. In particular, shock S3 is not observed by ISEE-3. What happened to it? Recall that S3 was advancing into the ejecta E2. One possibility is that this shock decayed because its speed relative to the ejecta dropped below the local magnetosonic speed. Figure 5 supports this conjecture. It shows by the black symbols the local magnetosonic speed on 4-5 April 1979. The magnetosonic speed is unusually high because of the strong magnetic field. The red symbols show the local speed of the shock relative to the ejecta E2, and one can see from the crossovers that the magnetosonic Mach number drops below unity at some point.

\subsection{Analysis of shocks at ISEE-3}

Shock 2 which passed the ISEE-3 at $\sim 01: 12$ UT (5), 1979, is a consistent fast forward IP shock with a normal $\mathbf{n}_{2}=(-0.64$, $0.715,0.28)$ (GSE) and a speed of $690 \mathrm{~km} \mathrm{~s}^{-1}$ in a solar inertial frame. The shock compression ratio is greater than that of S2 at Helios-2 ( 2.8 in both $n_{p}$ and $\left.B\right)$, consistent with the derived orientation, which is oblique to the upstream IMF $\left(\theta_{\mathbf{B}, \mathbf{n}_{2}} \approx 48^{\circ}\right.$; see Fig. 1). The oblique nature of the shock S2 is consistent, in turn, with the lack of a spike in the energetic particle flux at S2 (Fig. 3, bottom panel; see for example, Reames et al., 1996; Lepping et al., 2001). The shock orientation and speed suggest a discontinuity advancing locally strongly eastward. This is suggestive of a high solar wind gradient in the flow of matter away from the Sun in the neighborhood of S2. This shock is locally driven, as suggested by the observed speed at ISEE-3 of the leading edge of E2 at $\sim 10: 00$ UT (5), which is $\approx 700 \mathrm{~km} \mathrm{~s}^{-1}$. The orientation of this shock is consistent with a solar source on the west of the Sun.

3.5 Timing relationships between Helios-2 and ISEE- 3 ob-
servations

If the ejecta driving the shock S2 at ISEE-3 is the same ejecta E2 seen at Helios-2 on 4 April, it follows that its speed has increased on its way from Sun to Earth, i.e. it is not pristine at ISEE-3. This is possible if the faster ejecta E3 overtook 


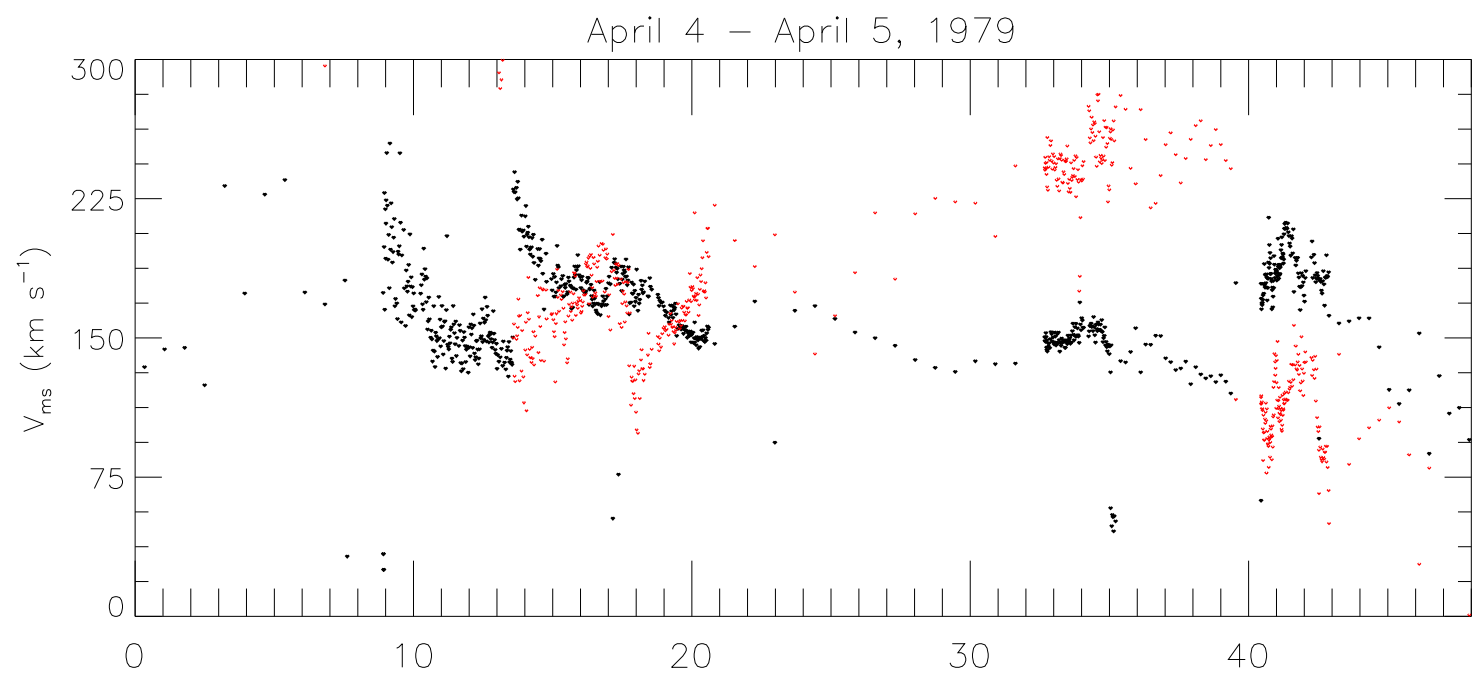

Fig. 5. The local magnetosonic speed on 4-5 April 1979 (black symbols) and the local speed of the shock S3 relative to the ejecta E2 (red symbols).

and coalesced with E2, (see also Sect. 2.1), thus increasing the speed of the leading ejecta E2. Compared to the pristine nature of E2 and E3 at Helios-2, an ejecta merger has taken place accompanied by an acceleration of E2 and a deceleration of E3.

In view of the longitudinal separation of Helios-2 and ISEE-3, which leaves open the possibility that the ejecta seen at Helios-2 does not extend as far as ISEE-3, we wish to support the above idea further. It may first be noted that for the entire ejecta interval 08:00 UT (5)-15:00 UT (6), shown in Fig. 3, there are two energetic particle hindrance regions (Forbush decreases), marked by arrows. These hindrances are also present at the start of each of the two pristine ejecta at Helios-2 (see arrows in Fig. 2). Hindrances in energetic particles are a reliable indicator of the start of ejecta regions (e.g. Richardson, 1997, and Fig. 1 in Lepping et al., 2001). This suggests that the two ejecta are seen at both locations. Assume now that the location of the second SEP decrease, which occurs at approximately 04:00 UT (6), represents the separatrix between the plasma of the coalesced transients E2 and E3. The speed at ISEE-3 of the middle point of E2 $\left(\sim 725 \mathrm{~km} \mathrm{~s}^{-1}\right.$ at 14:00 UT (5)) puts its lift-off at 08:00 UT (3). This is later than 00:00 UT (3), which is the start of the event predicted from Helios-2 observations, and which also lies within two hours of the time suggested by solar, radio and energetic particle data (Sect. 2.2). The shorter propagation time of $\mathrm{E} 2$ inferred from the later observations at ISEE-3 at a larger heliospheric distance implies that E2 is accelerated. To show that, at the same time, ejecta E3 is decelerated in the interaction, we take again its midpoint speed $\left(625 \mathrm{~km} \mathrm{~s}^{-1}\right.$ at 08:00 UT (6) at ISEE-3). This gives a lift-off time of 14:00 UT (3), earlier than inferred from the observations at Helios-2 of E3, which predict its lift-off at 10:00 UT (4) (Sect. 2.2), implying deceleration. According to this scenario, the deceleration of $\mathrm{E} 3$ is stronger than the acceleration undergone by E2.
We can attempt to obtain a rough estimate of the average size of the acceleration/deceleration. For this we use $<a>=\left(<V_{\text {app }}>-V_{t r}\right) / T$, where $<a>$ is the average acceleration/deceleration; $<V_{\text {app }}>=$ the apparent transit speed (the speed measured at the spacecraft); $V_{t r}$ is the actual transit speed, and $T$ is the time from launch to observation. For E2, we have $<V_{\text {app }}>=725 \mathrm{~km} \mathrm{~s}^{-1}$, $V_{t r}=480 \mathrm{~km} \mathrm{~s}^{-1}$, the latter obtained by dividing the radial distance of ISEE-3 by the time elapsed since launch, where launch time is inferred from Helios- 2 where the ejecta is pristine; and $T=86 \mathrm{~h}$. This gives $<a_{2}>\approx 0.78 \mathrm{~m} \mathrm{~s}^{-2}$. Similarly, for E3 we have $<V_{\text {app }}>=625 \mathrm{~km} \mathrm{~s}^{-1} ; V_{t r}=905 \mathrm{~km} \mathrm{~s}^{-1}$, using the lift-off time for the pristine ejecta, as derived from Helios-2=10:00 UT (4), and $T=46 \mathrm{~h}$. This implies $<a_{3}>\approx-1.8 \mathrm{~m} \mathrm{~s}^{-2}$. Assuming an elastic collision, this gives a rough estimate for the relative masses of the ejecta, $M_{2} / M_{3}=a_{3} / a_{2} \approx 2.3$.

Heating of the ejecta plasma also occurs as the shock S3 propagates through the preceding ejecta. At Helios-2, namely, there are strong gradients in $T_{p}$ : a factor of 10 frontto-back drop in the original E2, and a factor of 300 in E3. In contrast, at ISEE-3, the merged ejecta has a fairly uniform temperature. This average temperature $(\sim 70000 \mathrm{~K})$ is higher at the trailing end and somewhat lower than the leading edge of the merged ejecta. We take this to be evidence of heating, with possibly a concomitant heat exchange from the warmer E2 to the colder E3.

To summarize this section, by comparing with the propagation properties of pristine ejecta, we have produced evidence of acceleration of the leading ejecta and the deceleration of the trailing ejecta. The shock originally driven by the trailing ejecta has disappeared, and there is heating of both ejecta. The proton temperature in the coalesced ejecta E2-E3 is nevertheless still lower than that expected for normal solar wind expansion at $1 \mathrm{AU}$. 


\subsection{The magnetic cloud}

We first consider shock $\mathrm{S} 1$ ahead of the magnetic cloud E1. It passed Helios-2 at a radial distance 0.68 AU at 20:11 UT (2), 1979. Our method gives a shock normal $\mathbf{n}_{1}=(-0.97,-0.13$, $-0.24)$ in SE coordinates and a shock speed of $523 \mathrm{~km} \mathrm{~s}^{-1}$ in a solar inertial frame. The plasma is strongly compressed by the shock (by a factor $\sim 3$ ), but the compression in the magnetic field is weak. This is because the shock is quasiparallel $\left(\theta_{\mathbf{B}, \mathbf{n}} \approx 9^{\circ}\right)$. The shock orientation and speed suggest a shock traveling locally radially away from the Sun. It is possibly driven locally by the leading edge of the magnetic cloud E1, whose speed $\left(\sim 500 \mathrm{~km} \mathrm{~s}^{-1}\right)$ at $\sim 01: 12$ UT (3) is comparable to the speed of S1. The middle point of the magnetic cloud passes Helios-2 at approximately 08:00 UT (3) and, assuming the magnetic cloud to be pristine at the spacecraft location, its midpoint speed $\left(430 \mathrm{~km} \mathrm{~s}^{-1}\right)$ would give a transit time of $\sim 64 \mathrm{~h}$, placing its launch at $\sim 16: 00 \mathrm{UT}$ (31).

Shock S1 passed ISEE-3 at 09:22 UT (3). We found a consistent Rankine-Hugoniot solution with a shock normal $\mathbf{n}_{1}=(-0.92,-0.38,-0.40)$ in SE coordinates and a shock speed of $653 \mathrm{~km} \mathrm{~s}^{-1}$ in a solar inertial frame. The shock compression ratio is weak-to-moderate $\left(\sim 1.7\right.$ in $B$ and $\sim 2$ in $\left.n_{p}\right)$ and the shock is oriented obliquely to the upstream IMF, with $\theta_{\mathbf{B}, \mathbf{n}} \approx 54^{\circ}$. The shock orientation and speed suggest locally a shock inclined to the radial direction from the Sun. It is possibly driven locally by the leading edge of the magnetic cloud which, when it passed ISEE-3 at $\sim 20: 56$ UT (3), had a speed of $600 \mathrm{~km} \mathrm{~s}^{-1}$, i.e. the speeds match well.

Is the magnetic cloud E1 taking part in the ejecta merger? The "middle point" of the magnetic cloud passes ISEE-3 at approximately 10:00 UT (4). Using the assumption of a pristine ejecta, its midpoint speed $\left(467 \mathrm{~km} \mathrm{~s}^{-1}\right)$ places its launch at $\sim 17: 00$ UT (31), i.e. just 1 hour later than that inferred from Helios-2, and corresponding to a transit time of $89 \mathrm{~h}$. The agreement on the inferred launch times of the magnetic cloud from the two widely separated locations is very good and suggests strongly that E1 is pristine at both locations. It seems that the magnetic cloud is not participating in the merger (yet).

In agreement with the lift-off time of ejecta E2, several observatories record an $\mathrm{H} \alpha$ flare at S24 E21 with importance -B from 16:55 to 17:55 UT on 31 March. For that interval there is an LDE in the Soft X-rays (see Fig. 4). In addition, the Solar Maximum Mission coronograph lists a CME observation at 17:08 UT on 31 March with a speed in the plane of the sky of $408 \mathrm{~km} \mathrm{~s}^{-1}$, moving toward Earth. (Burlaga et al. (1987; Sect. 2.1) connect their magnetic cloud to the $\mathrm{H} \alpha$ flare from the same region at 23:15 to 23:55 UT on 31 March, slightly different from us.)

\section{Observations in March-April 2001}

The second example confirms the ejecta-ejecta signatures discussed above and illustrates other IP aspects of the coalescence of ejecta. We use data from the SOHO and Wind spacecraft during the period March-April 2001, with special emphasis on 28-31 March 2001.

Plasma and field observations from the SWE (Ogilvie et al., 1995) and MFI (Lepping et al., 1995) instruments on Wind are displayed in Fig. 6. Wind was executing a distant prograde orbit (DPO) and was located on average at (5, $-250,0) R_{E}$ (GSE coordinates).

The panels show from top to bottom the proton density, bulk speed, temperature and dynamic pressure (based only on the protons), the components of the magnetic field in GSE coordinates, the total field, the proton $\beta_{p}$, and the $\alpha$-to-proton number density ratio. (The latter quantity is from the ACE spacecraft. A propagation delay time of $1 \mathrm{~h}$ from ACE to Wind has been taken into account.) The red trace in panel 3 gives the expected solar wind temperature (after Lopez, 1987). Using the same criteria to identify ejecta as those employed in the first interval, we note a repetitive sequence of these transients: 7-8 may be identified in a 28 -day period. Several of these ejecta drove strong shocks, identified by a gradual rise in SEP fluxes as particles are energized at the travelling shocks (Cane et al., 1988). These data are not shown here (but see Berdichevsky et al., 2003). SEP onsets are concurrent with complex type III radio bursts observed by Wind/WAVES (Bougeret et al., 1995). Correlated with this combination of ejecta and driven shocks, a sawtooth V-profile with an increasing tendency (blue trace) from 28 March to 14 April $\left(\sim 550\right.$ to $\left.\sim 820 \mathrm{~km} \mathrm{~s}^{-1}\right)$ is observed. In Sect. 4.5 this sequence of CMEs will be associated with a sequence of large, episodic enhancements of the terrestrial ring current.

We now focus on the 50-h interval 00:00 UT 30 March to 04:00 UT 1 April. Figure 7 plots the plasma, magnetic field, and energetic particle fluxes, in the same format as Fig. 2. The $2 \mathrm{MeV}, 8 \mathrm{MeV}$ and $20 \mathrm{MeV}$ fluxes in the bottom panel are from the Low Energy Matrix Telescope (LEMT), a component of the Energetic Particles Acceleration, Composition, and Transport (EPACT) Investigation on the Wind spacecraft (von Rosenwinge et al., 1995). The period divides neatly into two: the first part (30 March) is characterized by low velocity, low temperature, relatively low dynamic pressure, and a weak magnetic field, dropping on occasion to very low values $\sim 1 \mathrm{nT}$. In contrast, the second (31 March) is a region of high density, high velocity, high temperature, high dynamic pressure, a generally low $\beta_{p}$ (with brief excursions to higher values), and an extremely high magnetic field subject to largeamplitude variations. Three shocks are present, $S_{I}-S_{I I I}$. $S_{I}$ and $S_{I I I}$ bracket the region of interest. The low $\beta_{p}$, strong fields, and a generally high $n_{\alpha} / n_{p}$ number density ratio characterizing this interval between $S_{I I}$ and $S_{I I I}$ indicate ejecta material. Just behind $S_{I I}$, extremely high dynamic pressure values are reached ( $\sim 100 \mathrm{~Pa}$; based just on the protons). Above average ejecta densities $\left(<n_{p}>=22.0 \pm 22.1 \mathrm{~cm}^{-3}\right)$ and speeds $\left(<v_{p}>=633.0 \pm 46.1 \mathrm{~km} \mathrm{~s}^{-1}\right)$ are observed in the interval bounded by shocks $S_{I I}$ and $S_{I I I}$.

Closer inspection of the 31 March interval reveals a set of concurrent disturbances at 12:00-14:00 UT: a spike in $\beta_{p}$ to values above unity, a decrease in $B$, and a north-south 


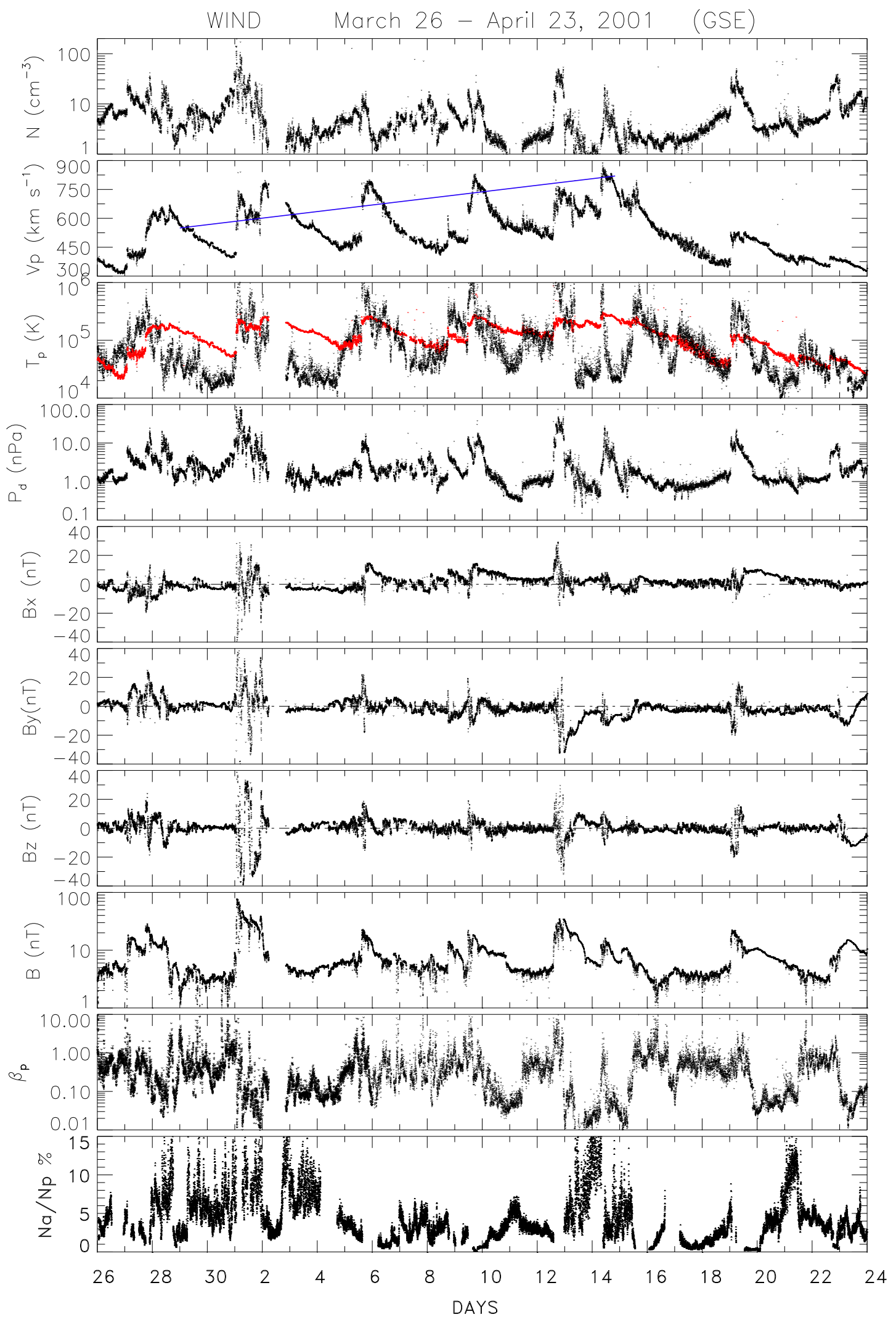

Fig. 6. Plasma and magnetic field data from the Wind spacecraft for the time interval 26 March-24 April 2001. From top to bottom are plotted the proton number density, bulk speed, temperature and dynamic pressure, the components of the magnetic field in GSE coordinates, the field strength, the proton plasma beta and the $\alpha$-particle to proton number density ratio in percent. 


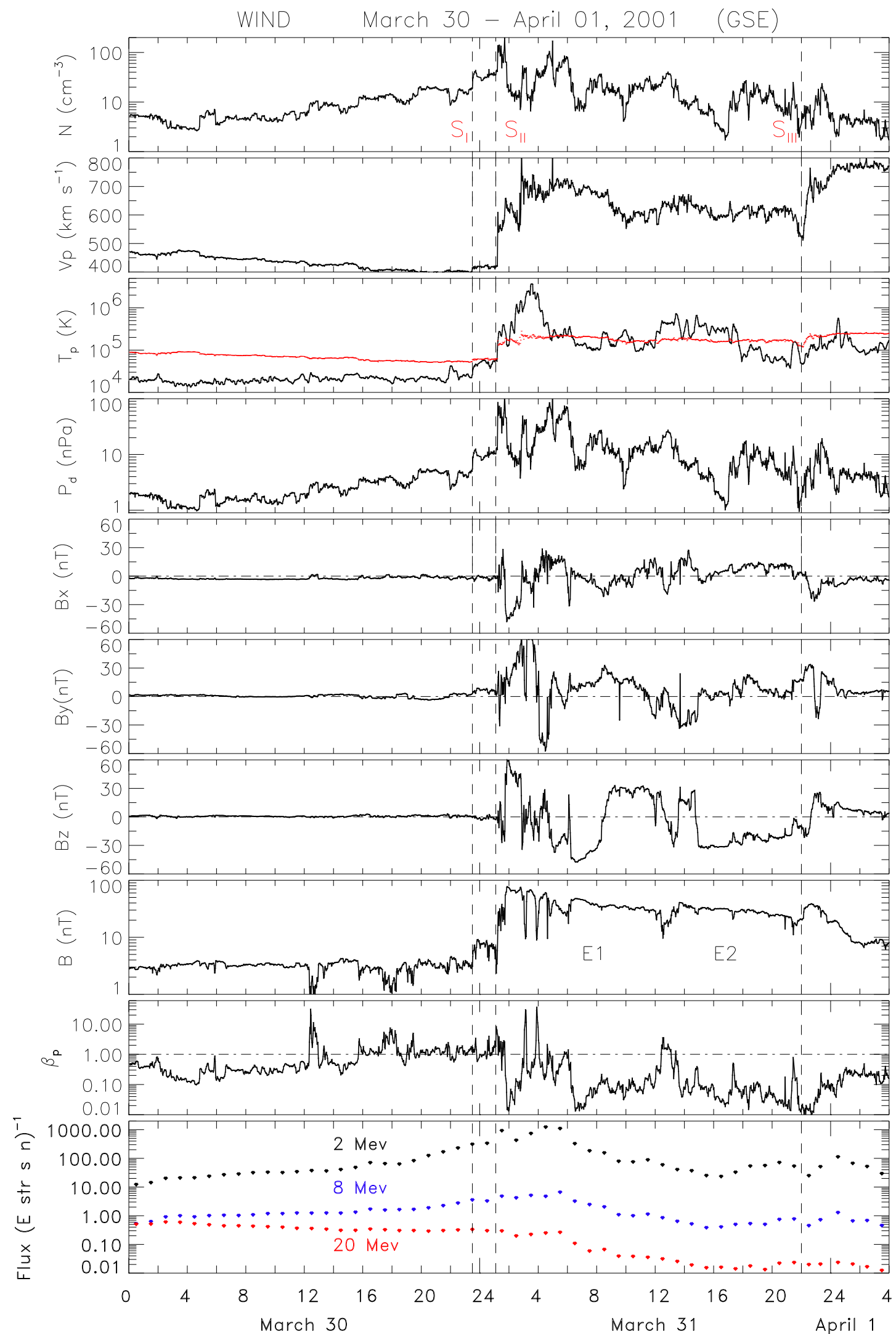

Fig. 7. A blow-up of the period 30 March-04:00 UT, 1 April showing proton plasma, magnetic field and energetic particle data. The figure highlights the differences in plasma and field parameters measured on 30 March and 31 March, respectively. 
Table 2. Similar to Table 1, but for the shocks in the March 2001 event. Vector quantities are given in GSE system. $S_{I}$ is evaluated using Wind data, and $S_{I I}$ is evaluated using ACE data.

\begin{tabular}{llccrccccccc}
\hline No. & $\begin{array}{l}\text { Day } \\
\text { April }\end{array}$ & $\begin{array}{l}\text { Time } \\
(\mathrm{UT})\end{array}$ & Normal & $\begin{array}{r}V s^{\prime} \\
\mathrm{km} \mathrm{s}^{-1}\end{array}$ & $\begin{array}{l}V s \\
\mathrm{~km} \mathrm{~s}^{-1}\end{array}$ & $\begin{array}{l}\Theta_{B, n} \\
\left({ }^{\circ}\right)\end{array}$ & $B_{d w} / B_{u p}$ & $n_{d w} / n_{u p}$ & $M s$ & $\begin{array}{l}\text { SC } \\
(\mathrm{AU})\end{array}$ & $\begin{array}{l}\text { Loc. } \\
\left({ }^{\circ}\right)\end{array}$ \\
\hline WIND & & & & & & & & & & & \\
$I$ & 30 & $23: 30$ & $(-0.59,-0.57,-0.57)$ & 59 & 438 & $83 \pm 1$ & $1.3 \pm 0.2$ & $2.0 \pm 0.3$ & 1.2 & 0.99 & $0 \mathrm{E} 0 \mathrm{~S}$ \\
$I I$ & 31 & $00: 23$ & $(-0.74,-0.42,-0.50)$ & 197 & 597 & $66 \pm 10$ & $2.2 \pm 0.2$ & $4.0 \pm 0.2$ & 3.6 & 0.99 & $0 \mathrm{E} 0 \mathrm{~S}$ \\
\hline
\end{tabular}

rotation of the magnetic field occurring in an increasing solar wind speed recovering from a large depression $\left(\sim 100 \mathrm{~km} \mathrm{~s}^{-1}\right)$, and an interruption in bidirectional streaming of $166 \mathrm{eV}$ solar wind halo electrons monitored by the 3-D Plasma Analyzer on Wind (Lin et al., 1995; data not shown). We shall propose that these signatures mark the remnants of the boundary between two ejecta (called E1 and E2 in the figure), E1 being faster and having a stronger field than E2. Below we shall support this idea by discussing solar observations (We note that Sun et al. 2002, see Sect. 2.1, misidentified the second ejecta as a corotating interacting region; see their Fig. 2.). We discuss this further in Sect. 4.4.

\subsection{Analysis of shocks at wind}

We now analyze the shocks (See also Table 2.). For $S_{I}$, which passed Wind at 23:30 UT on 30 March 2001, we obtain a shock normal $\mathbf{n}_{I}=(-0.59,-0.57,-0.57)$ in GSE coordinates, and a speed of $438 \mathrm{~km} \mathrm{~s}^{-1}$ in a solar inertial frame. The shock compression ratio is $\sim 2$ in both $B$ and $n_{p}$, and the shock is quasi-perpendicular $\left(\theta_{\mathbf{B}, \mathbf{n}_{\mathbf{I}}} \approx 83^{\circ}\right)$. The shock orientation and speed suggest a disturbance that is locally quite inclined to the radial direction from the Sun (Usually such inclined shocks are not locally driven, as pointed out in Berdichevsky et al., 2001.). It could be that this shock is distorted after it traversed the ejecta E1 ahead of it (Such distortions also result from simulations, see Sect. 5.3.).

The passage of $S_{I I}$ at Wind at 01:12 UT, 31 March occurs at/near a strong dip in the magnetic field (magnetic hole), which makes it difficult to evaluate the shock properties at this spacecraft. We use ACE data instead. We obtain a shock normal $\mathbf{n}_{\text {II }}=(-0.745,-0.428,-0.50)($ GSE) traveling at a speed of $597 \mathrm{~km} \mathrm{~s}^{-1}$ in a solar inertial frame. Unlike $S_{I}, S_{I I}$ is a very strong shock ( $\sim 4$ compression in $n_{p}$ and $\sim 3$ in $B$ ) and is quasi-perpendicular $\left(\theta_{\mathbf{B}, \mathbf{n}_{2}} \approx 66^{\circ}\right)$. This second shock speed matches the local speed of the ejecta better.

We now discuss what the lift-off times of E1 and E2 would be if we were to assume pristine conditions. Ejecta E1 appears to commence its passage at Wind somewhere between 04:00-06:00 UT, 31 March. Its midpoint passes Wind at approximately 07:00-09:00 UT, 31 March, with a speed of $\sim 700 \mathrm{~km} \mathrm{~s}^{-1}$. If it were pristine, the corresponding CME would have lifted off the Sun $\sim 60 \mathrm{~h}$ earlier, i.e. at 2000 $\pm 01: 00$ UT, 28 March. Ejecta E2 appears to start its passage at Wind at $\sim$ 13:00 UT and continues until about 22:00 UT when $S_{I I I}$ is observed. Its middle point passes Wind at $\sim 16: 30$ UT with a speed $\sim 610 \mathrm{~km} \mathrm{~s}^{-1}$. If it were a pristine ejecta, this would place its lift off $68 \mathrm{~h}$ earlier, at 20:30 UT on 28 March, i.e. very close to the lift-off time of ejecta E1 assuming pristine conditions.

To summarize this section so far: two ejecta are seen by Wind behind $S_{I I}$ in an advanced stage of coalescence. The interaction has caused a very strong shock in front of the leading ejecta, very compressed magnetic field strengths (with, in particular, large, out-of-the-ecliptic components), and high plasma densities, and has heated the plasma, the latter indicated by the high values shown in the second panel of Fig. 7, which are comparable to those expected for normal solar wind expansion. We also obtained the launch times for the individual ejecta we would obtain if they were pristine. These are certainly wrong, as the solar observations discussed next show.

\subsection{Solar observations}

Relevant data for 28-30 March are shown in the top two panels of Fig. 8. The first panel displays the intensity of the $1-8 \AA$, and $0.5-4 \AA$ soft X-ray solar radiation measured by the environmental satellite GOES 8 . The repeated flaring is indicated by repeated enhancements in this radiation. The middle panel shows the altitude-versus-time profile for the CMEs. The heavier traces refer to H-CMEs and they are the ones which concern us further below. The bottom panel presents energetic particle fluxes of energies $2 \mathrm{MeV}, 8 \mathrm{MeV}$ and $20 \mathrm{MeV}$, respectively, as measured by the LEMT instrument on Wind.

The first H-CME was identified as a likely backside event, and hence we concentrate here on H-CMEs two and three. (Hereafter we shall refer to these as H-CME 1 and H-CME 2 , respectively, since we associate them with ejecta $\mathrm{E} 1$ and E2, respectively.) H-CME 1, directed toward Earth, is seen at 2 solar radii at 13:27 UT on 28 March, and appears to be related to the 12:50 UT, soft X-ray flare M4.3, LDE, with possible $\mathrm{H} \alpha$ flare signature at $\mathrm{N} 18 \mathrm{E} 02$. H-CME 2 is seen at 2 solar radii at 10:20 UT on 29 March. This H-CME is related to the 10:15 UT soft X-ray flare X1.7, LDE, with possible optical $\mathrm{H} \alpha$ flare signature at N20W18, and same region EIT/SOHO brightening in the Fe XII line. These sources agree with the identifications of Sun et al. (2002), see Sect. 2.1. As panel 3 shows, H-CME 1 is related to 

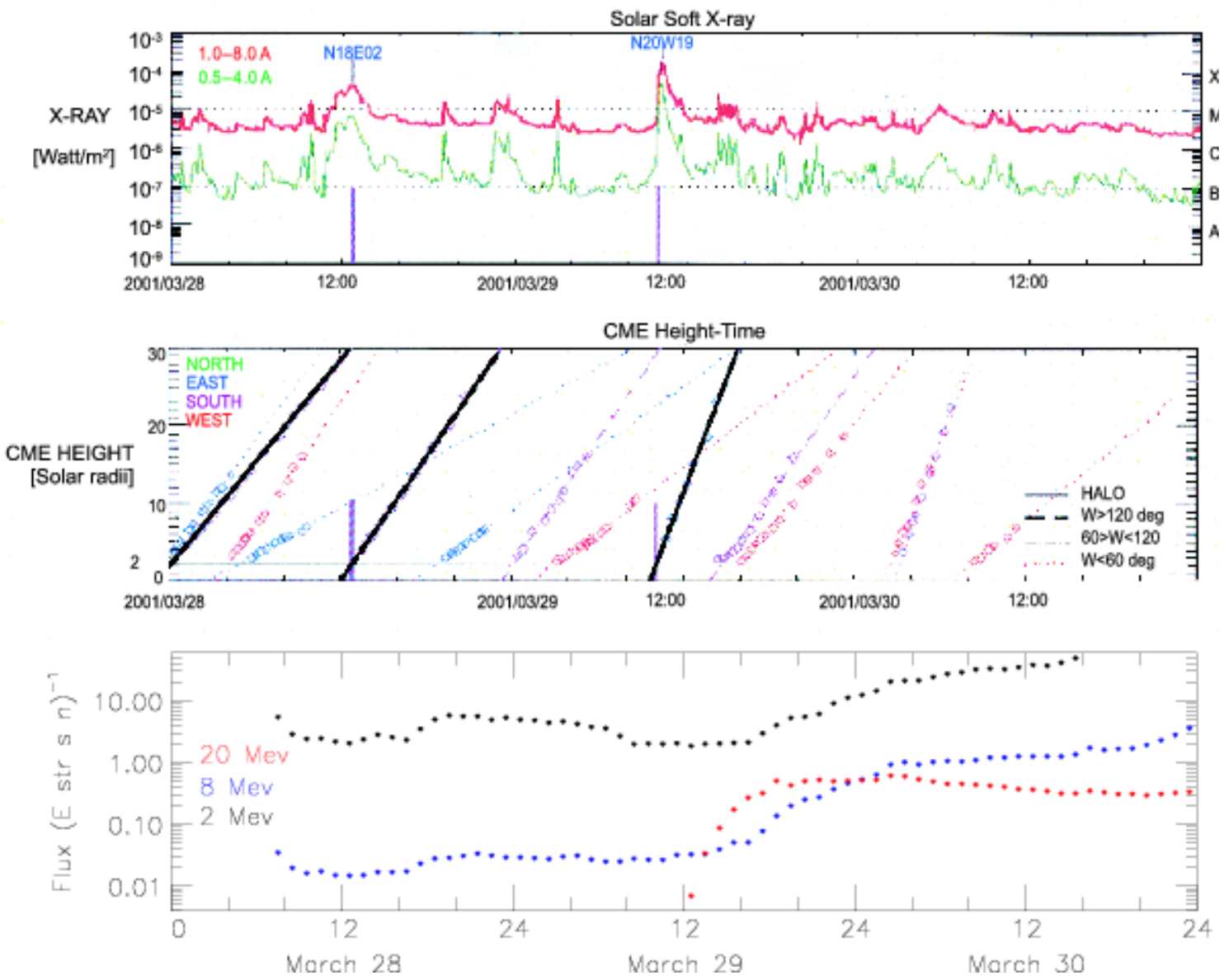

Fig. 8. Solar observations (top panels). Bottom panel: LEMT/EPACT measurements of high energy proton fluxes. A dispersional injection is seen starting at $\sim 12: 00 \mathrm{UT}, 29$ March in the highest energy panel.

the dispersive impulsive onset observed by LEMT, starting at about 12:00 UT in the $20 \mathrm{MeV}$ channel on 29 March. This allows about $2 \mathrm{~h}$ for these particles to reach Wind, a reasonable estimate.

The middle panel in Fig. 8 indicates a progressively steeper slope with increasing time for the H-CMEs. Linearly extrapolating the altitude-versus-time plots shown for H-CME 1 and 2, and assuming no interactions, we would conclude that H-CME 2 overtook H-CME 1 at $\sim 07: 40$ UT on 30 March near $0.56 \mathrm{AU}$.

\subsection{Timing relationships between IP and solar observations}

The observed coronagraph launch time for H-CME 1 is earlier than that inferred in the previous subsection (13:27 UT versus 20:00 UT, 28 March), i.e. it must have moved slower in the inner heliosphere than at $1 \mathrm{AU}$, i.e. it has accelerated. The converse is true for H-CME 2 (10:20 UT, 29 March versus 20:30 UT, 28 March); it has slowed down. Figure 8 yields speeds in the plane of the sky of $\sim 535 \mathrm{~km} \mathrm{~s}^{-1}$ and $\sim 1070 \mathrm{~km} \mathrm{~s}^{-1}$ for H-CME 1 and H-CME 2, respectively, so they are approaching each other within $30 R_{S}$ at a relative speed of $535 \mathrm{~km} \mathrm{~s}^{-1}$. Because velocities in the plane of the sky are a lower limit to the actual speed of the ejecta, we consulted the available radio information on metric and IP radio signatures of the speed of the driven shock(s). There are no metric radio emissions during the lift-off of the H-CME 1. There is a patchy signature of its driven shock in Wind/WAVES decametric data only (M. Kaiser, private communication, 2001). For H-CME 2, there are signatures, too complex to interpret, which extend from about launch to local passage of the shock $S_{I I}$ and beyond. On 29 March 2001 IZMIRAN radio observers identified metric Type II radio bursts from 10:04 UT (145 MHz) to 10:08 UT (78 MHz), suggesting an estimated CME propagation (shock speed close to the base of the corona) with a speed of $1300 \mathrm{~km} / \mathrm{s}$. This shock can be associated with the N20W19 X1.7 SF flare from AR 9402, on 29 March 2001. However, the inferred IZMIRAN radio signal speeds do not imply a much faster motion than the plane-of-sky values obtained by LASCO/SOHO.

We now estimate the relative masses of ejecta 1 and 2 . For ejecta 1 we have $V_{\text {app }}=700 \mathrm{~km} \mathrm{~s}^{-1}$ and from the launch time, 13:27 UT (28), and its observation time at $1 \mathrm{AU}$, $\sim 08: 00$ UT (31), we have $V_{t r}=627 \mathrm{~km} \mathrm{~s}^{-1}$, and $T=66.5 \mathrm{~h}$. This gives $<a_{1}>\approx 0.5 \mathrm{~m} \mathrm{~s}^{-2}$. Similarly, for ejecta 2 , we have 


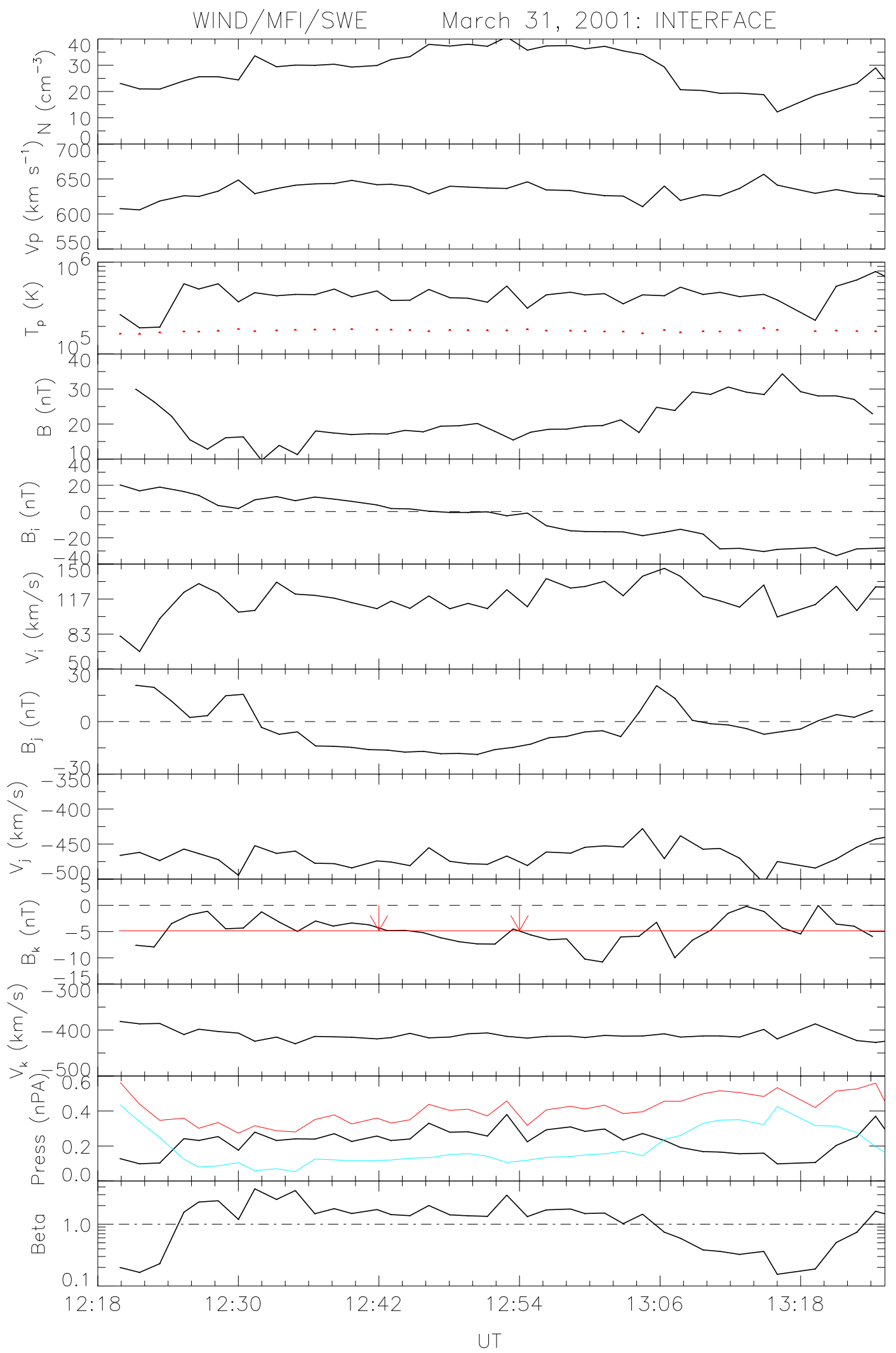

Fig. 9. The interface between ejecta 1 and 2. The panels show from top to bottom the proton density, bulk speed and temperature, (pairwise) components of field and flow in principal axes coordinates $(\mathrm{i}, \mathrm{j}, \mathrm{k})$; the pressures (blue: magnetic field, black: plasma (electrons plus protons) and red: their sum), and the proton beta. 
$V_{\text {app }}=610 \mathrm{~km} \mathrm{~s}^{-1}, V_{t r}=769 \mathrm{~km} \mathrm{~s}^{-1}$, and $T=54.2 \mathrm{~h}$, yielding $<a_{2}>\approx-0.81 \mathrm{~m} \mathrm{~s}^{-2}$. An estimate of the ratio of the masses is then $M_{1} / M_{2} \approx 1.6$.

To sum up, based on H-CME observations and metric type II radio signals we obtained CME speed estimates at the Sun to infer possible interactions. We found that H-CME 2 caught up with and compressed CME 1 . The mass of ejecta 1 is estimated as 1.6 that of ejecta 2, consistent with the larger densities in E1 seen at Wind. At Wind, the IP manifestations of the merger are a very strong shock driven by the leading ejecta, a dense and hot plasma, and compressed $>30 \mathrm{nT}$ field regions following in quick succession. They are separated by a set of disturbances marking an interface. We discuss this next.

\subsection{The boundary between ejecta 2 and 3}

The major problem in investigating the boundary is that we do not know exactly where it starts and where it ends. We shall assume a conservative estimate and let it be defined by the criterion $\beta \sim 1$. This then corresponds to the interval 12:20-13:25 UT (31). We carried out a minimum variance analysis of this interval (Sonnerup and Cahill, 1967). The routine picked out a very well-defined plane (ratio of intermediate-to-minimum eigenvalues $=20$ ), whose normal is $\mathbf{k}=(0.67,0.72,0.01)$. The magnetic field normal to the plane is $B_{k}=5.4 \pm 3.5 \mathrm{nT}$. The period is shown plotted in principal axes coordinates (ijk) in Fig. 9. The panels are, from top to bottom, the proton density, bulk speed and temperature, (pairwise) components of field and flow in principal axes coordinates $(i, j, k)$; the pressures (blue: magnetic field, black=plasma (electrons plus protons) and red is their sum), and the proton beta.

We may note the following: (i) a planar sheet in approximate pressure balance (panel 11) separates the two ejecta; there is evidence of (ii) a depression in the magnetic field (panel 4); (ii) heating of the plasma (panel 3); and (iv) a non-zero normal field component $B_{k}$ (panel 9, red line). All of these are consistent with the boundary being a rotational discontinuity and with ongoing reconnection. However, if we assume a 1-D, time-independent structure, and check for the conservation of momentum tangential to the boundary, i.e. $\Delta \mathbf{v}_{t}=\alpha \Delta\left(\mathbf{B}_{t} / \rho\right)$, (where $\rho$ is the mass density, $\alpha$ is a pressure anisotropy factor $\left.\left(=\left(p_{\|}-p_{\perp}\right) \mu_{0} / B^{2}\right)\right)$, suffix " $\mathrm{t}$ " denotes quantities along the plane with $\Delta$ meaning values through the boundary relative to a reference point, Sonnerup et al., 1981)), we run into a second problem: where is the quiet reference? Forming averages over the $15 \mathrm{~min}$ prior to the interval shown in Fig. 9, we obtain quantities, some of which have large error bars (standard deviation). Using these, we obtain correlation coefficients of -0.2 (in $i$ components) and 0.3 (in $j$ components), an inconsistent result. For this reason we consider the evidence for reconnection at the boundary between the ejecta to be poor. The lack of a convincing rotational discontinuity may be due to the low magnetic shear across the boundary, $60^{\circ}$ in this case (We tried other intervals, and different reference positions, but while
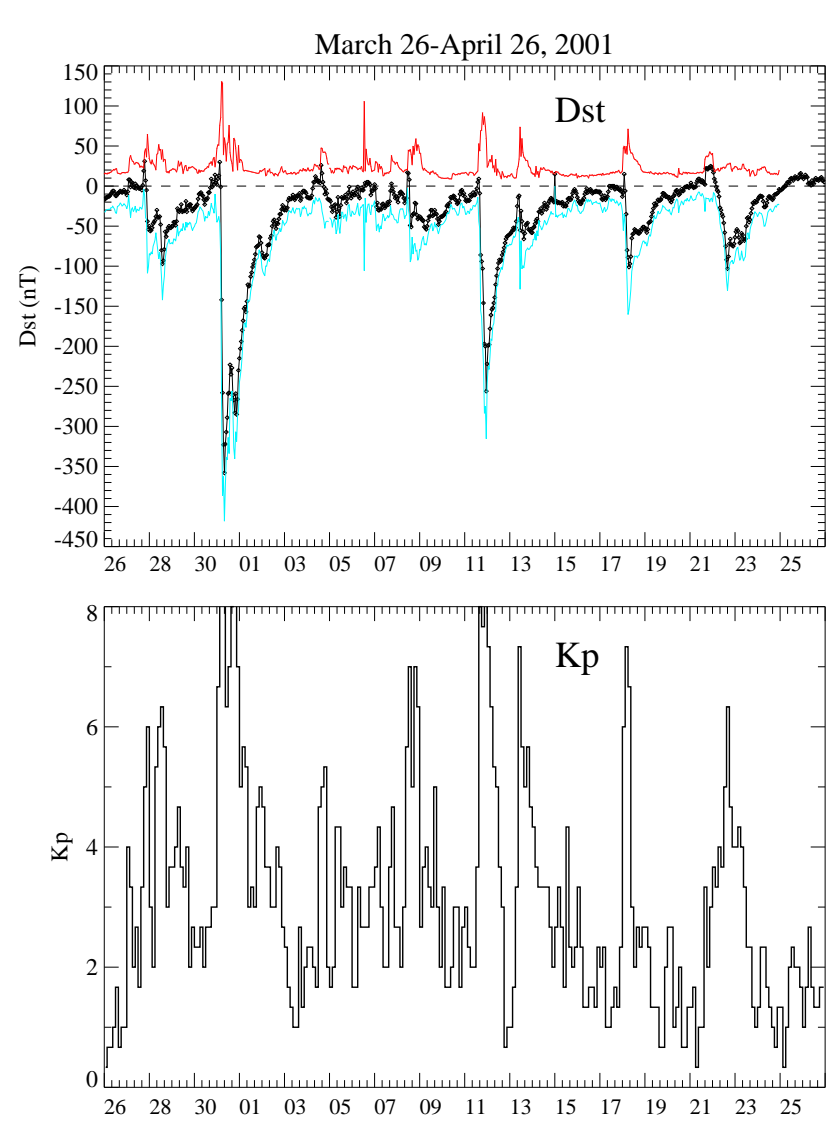

Fig. 10. $K_{p}$ and $D_{s t}$ measurements for 26 March- 26 April 2001. The effect of the $\alpha$-particles in calculating the magnetopause currents (top red trace) has not been taken into account. For further details, see text.

the results are quantitatively different, they are the qualitatively similar.).

\subsection{Contrasting the geoeffects of the two intervals: $D_{s t}$ and $K_{p}$}

One reason for studying ejecta-ejecta interactions is the geoeffects they elicit and how these differ from those of isolated ejecta. Here we study $D_{s t}$ and $K_{p}$ profiles, starting with the year 2001 interval. Figure 10 refers to the 1 -month period 26 March-26 April 2001. The format of this and the next two figures is as follows: the black trace in the top panel shows the measured $D_{s t}$ values. The red trace at the top is the disturbance of the horizontal component of the ground geomagnetic field caused by Chapman-Ferraro (magnetopause) currents. The blue trace is the $D_{s t}$ corrected for this effect $\left(D_{s t} *\right)$ and reflects better the enhancements of the terrestrial ring current. The bottom panel shows the 3-hourly $K_{p}$ index. To form an idea of the magnitude of the storms, we recall that storms whose peak $D_{s t} * \leq-100 \mathrm{nT}$ are classified as "major", while those with $D_{s t} * \leq-250 \mathrm{nT}$ are classified as "great" (e.g. Tsurutani et al., 1992).

The period shown in Fig. 10 was one of the most disturbed periods ever recorded, with two/three great storms 

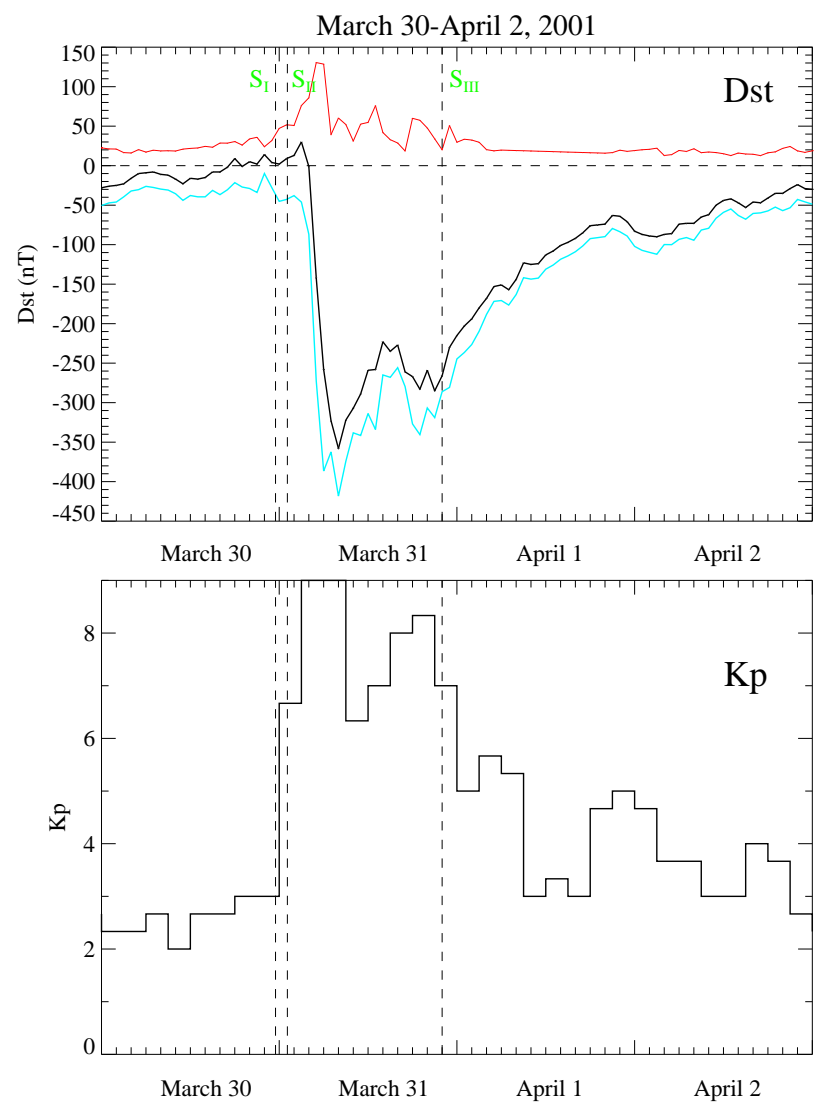

Fig. 11. Similar to Fig. 10 but for the shorter interval 30 March-2 April.

$\left(D_{s t}<-250 \mathrm{nT}\right)$ and 5 other major storms $\left(D_{s t}<-100 \mathrm{nT}\right)$. Repeated flaring was seen at the Sun (see Fig. 8). A comparison with Fig. 6 shows that there is practically a 1-1 correspondence of ejecta and episodic ring current enhancements. Therefore, the active period at the Sun produces a similarly active period on Earth.

We focus now on one of the largest storm in this period. This occurred on 31 March, during the passage of the two interacting ejecta E1-E2. Does the ongoing ejecta merger leave an imprint on the ground magnetic field? Figure 11, covering the 4-day interval 30 March-2 April 2001, shows that in fact it is a double-dip storm. The storm reaches a peak value of $-420 \mathrm{nT}$ during the passage of the compressed-field of E1, with its large negative $B_{z}$ due to the compression of the magnetic field and the plasma by the ejecta-ejecta interaction (see Fig. 7). Its recovery is then momentarily halted (at a northward magnetic field turning) and reversed when the trailing ejecta E2 arrives. This is a double great storm occurring in less than one day. The $D_{s t} *$ remained $\leq 250 \mathrm{nT}$ for $\sim 20 \mathrm{~h}$. One effect of the compressed plasma may be seen by the large contribution of the magnetopause currents. Alone during the main phase, they contribute $\sim 130 \mathrm{nT}$ to the ground field disturbance. Worth noting also is the saturation of the $K_{p}$ index, which recurs 12 days later (Fig. 10) in the third great storm in April 2001.
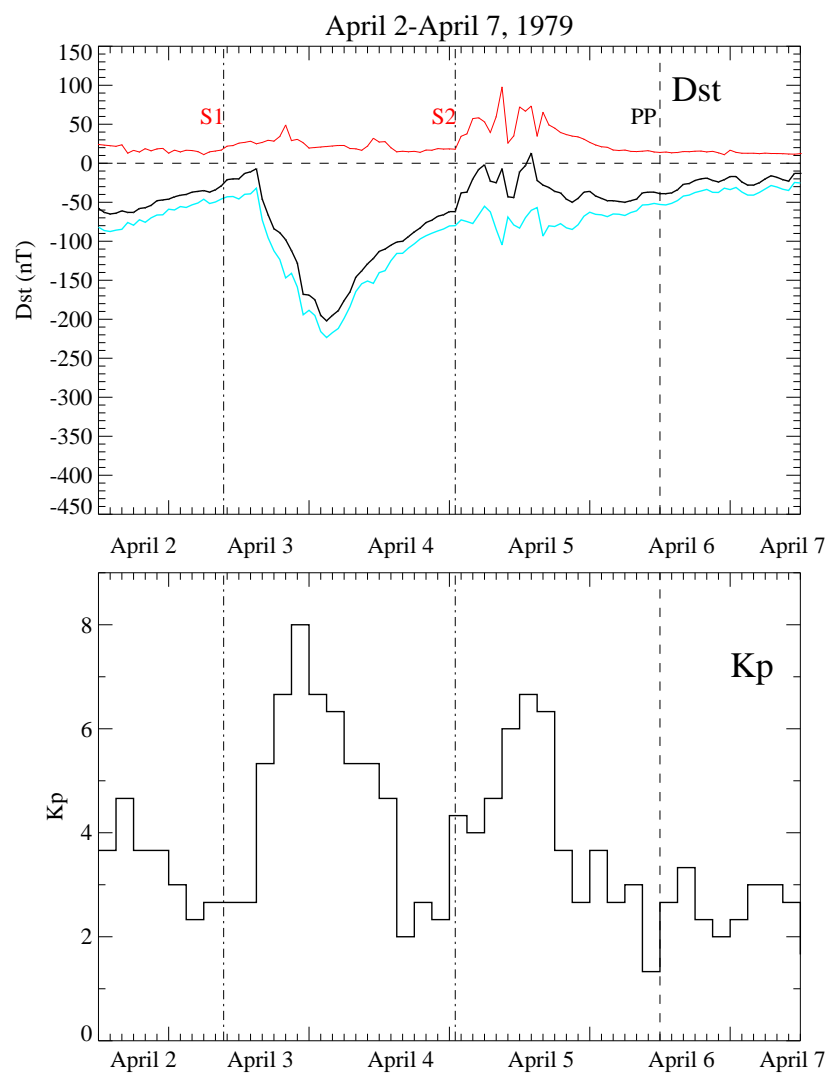

Fig. 12. Similar to Fig. 10, but for the first event sequence. The period plotted is 2-7 April, 1979.

For contrast, we now show similar results for 2-7 April 1979 which, we recall, contained a non-interacting magnetic cloud followed by a merger of two ejecta. From Fig. 12, two surges of activity are apparent. The first (and largest) is due to the magnetic cloud which, as seen in Sect. 3.6, was not participating in the merger (i.e. was pristine both at Helios-2 and at ISEE-3). The complex ejecta later produced little in the way of $D_{s t}$ intensification. Yet $D_{s t} *$ went below $-100 \mathrm{nT}$. This was, however, mainly due to an enhancement of the magnetopause current, i.e. to dynamic pressure brought about, in part, by plasma compression during the interaction (Fig. 2). Paradoxically, therefore, and in contrast to March 2001, the main effect on the ground was due to the non-participating member of the multiple ejecta, and one effect of the interaction (plasma compression) accounted for part of the rest of the $D_{s t} *$, the other part being due to enhanced dynamic pressure in the sheath.

\section{Summary and discussions}

\subsection{Summary}

We have examined two IP data intervals separated by two solar cycles and each near solar activity maximum, where the twin-spacecraft observations indicate ejecta interacting 
with each other to form, in the terminology of Burlaga et al. (2001), complex ejecta. Our aim was to isolate observational IP signatures of ejecta-ejecta interactions. These intervals have been examined by other people (see Sect. 2.1), but we have added further extensive analysis and quantitative estimates with the following results. In both event sequences, ongoing coalescence/merging was accompanied by (i) acceleration of the leading ejecta; (ii) deceleration of the trailing ejecta; (iii) weakening and/or disappearance of shocks originally driven by the trailing ejecta; (iv) strengthening of shocks associated with the accelerated ejecta; (v) compression of plasma and (vi) magnetic field of the leading ejecta; and (vii) heating of ejecta plasma. We gave an estimate of the relative masses of the interacting ejecta. We also examined the interface between the interacting ejecta on March 2001 and found that a plane could be very well defined with a nonzero normal field component. Other tests for reconnection (stress balance analysis) gave a negative result, so that reconnection did not seem to be occurring. We ascribed this to the lack of sufficient magnetic shear across the boundary.

\subsubsection{Comparing the two active intervals}

At the Sun, between 30 March and 4 April 1979, we observe a very active interval with a soft $\mathrm{X}$-ray background radiance of $\sim 10^{-6} \mathrm{~W} \mathrm{~m}^{-2}$. During that interval, flares were identified at the rate of at least 30/day at longitudes within $40^{\circ}$ of central meridian. There were also large numbers of soft X-ray, LDEs, and about two CMEs/day observed with SMM, many metric radio emission bursts, including types IV and II, indicative of fast, solar front-sided, propagating CMEs.

Similar features on the Sun's disc are observed in 28-30 March 2001. There is, in fact, a larger number of CME observations (see, e.g. Fig. 8). However, these more recent CME observations are performed using the coronagraph LASCO on the SOHO spacecraft, which has a higher cadence and is far more sensitive than any coronagraph previously flown (see e.g., Michels, 1998, and Berdichevsky et al., 2002). An outstanding difference is that the March-April 2001 period coincides with the passage of a complex solar active region containing the largest coronal sunspot system recorded in the 23 rd solar cycle.

There are also similarities in the $1 \mathrm{AU}$ observations. Consult Figs. 2 and 7.

1. Both intervals were bracketed by shocks and/or pressure pulses.

2. The bulk speed profiles both before and after the shocks are similar.

3. Before shocks $\mathrm{S} 2$ and $S_{I I}$ the IP medium is a cold and slow solar wind.

4. There are similar strengths in the SEPs (medium gradual SEP events).

5. Strong magnetic fields follow shocks $\mathrm{S} 2$ and $S_{I I}$.
6. High dynamic pressures and very hot plasmas follow these shocks.

7. Both events were cases of complex ejecta.

8. There is a similar two-step hindrance (Forbush decreases) at the interface of the coalescent ejecta present in both cases. This shows that the energetic particles still sense some vestige of the original individual ejecta.

Major differences are:

1. The orientation of the magnetic field in the 1979 event is mostly northward or $B_{z} \approx 0 \mathrm{nT}$, whereas in the 2001 event it was mostly southward.

2. Shock $S_{I I}$ in 2001 is stronger than S2 in 1979.

3. The strong shock $S_{I I}$ appears to be able to accelerate particles in the $\sim 2 \mathrm{MeV}$ range.

4. In April 1979, there was an ejecta clearly not participating in the merger.

5. In April 1979, one shock disappeared, whereas in 2001 both shocks are present, albeit $S_{I}$ is very weak.

6. The strength of the magnetic field (at $1 \mathrm{AU}$ ) is much higher in the 2001 event, representing an unusually high compression of the ejecta. In particular, this compression led to two epsiodes of large negative $B_{z}$ and a very intense two-humped $D_{s t}$ (see Sect. 5.4 below).

\subsection{Some implications of the analysis}

We now comment on some of the points raised above and their implications. The acceleration/deceleration of components of the ejecta merger (items $i$ and ii) stands in sharp contrast to the conclusion arrived at from studies of pristine ejecta (separated by 4 days) near the last solar minimum. A repetitive finding there was that ejecta tend to retain their speed in going from the Sun to the Earth (Berdichevsky et al., 2002).

The heating of ejecta plasma (item vii) is worth commenting upon (see also Sect. 2.1). In pristine ejecta, low proton temperatures (compared to those expected for normal solar wind expansion) are taken, rightly, to be a very robust signature of ejecta material in space (Gosling, 1990; Richardson and Cane, 1995, and references therein.) With the heating accompanying the interaction of ejecta, this identification signature become problematic. The proton plasma $\beta_{p}$ should be used instead. Because the field and plasma are compressed and the plasma is heated, the proton plasma $\beta_{p}$ in our examples tend to remain $\ll 1$.

An example of the disappearance of the shock originally driven by the trailing ejecta (item iii) was shock S3 in the April 1979 example. The disappearance of a shock and the transfer of the momentum of the post-shock flow to the leading magnetic cloud (see Sect. 5.3 and Odstrcil et al., 2003) removes one geoeffective element of isolated ejecta driving 
shocks. Past studies have shown that the shock and postshock flows can in themselves cause substantial geoeffects (Gosling et al., 1990, 1991).

An example of strengthening of the shock driven by the leading ejecta (item iv) is shock $S_{I I}$ arriving at Wind on early 31 March 2001. This strong shock has evidently developed the ability to energize particles in the $\mathrm{MeV}$ range (Fig. 7, bottom panel). Such energization at shocks may thus reflect an evolutionary trend. Clearly, this development is very important for the subsequent effect of the configuration on the geophysical environment, and would seem to be an element peculiar to ejecta-ejecta interactions.

The compression of the plasma (item v) is another such effect. In terms of geoeffectiveness, a compressed plasma leads to a large dynamic pressure - as seen in our examples - and hence to large magnetopause currents. These Chapman-Ferraro currents cause large disturbances which can reach values $\sim 100 \mathrm{nT}$ and thus affect the ring current substantially. Aside from this, large IP densities are believed to lead, under conditions not yet well understood, to a superdense plasma sheet $\left(n_{p}>1 \mathrm{~cm}^{-3}\right.$; Borovsky et al., 1998). A dense plasma sheet coupled to a strong convection electric field leads to strong ring currents (e.g. Jordanova, et al., 1998). Conversely, low plasma sheet densities are one element hastening the decay of the ring current (Jordanova et al., 2003).

The extraordinary strength of the magnetic field in ejecta 1 on 31 March (34.2 nT $\pm 12.5 \mathrm{nT})$ (major difference 6) may be gauged from a comparison with typical ejecta field strengths at $1 \mathrm{AU}$. Thus, a statistical study based on 30 magnetic clouds observed by Wind near solar minimum yields an average strength at $1 \mathrm{AU}$ is $13 \pm 1.0 \mathrm{nT}$ (Lepping et al., 2003). The average density and transit speed emerging from this study $=11.4 \pm 2.9 \mathrm{~cm}^{-3}$, and $396 \pm 16 \mathrm{~km} \mathrm{~s}^{-1}$ (mean \pm standard deviation). In all three parameters, then, 31 March represents a large deviation from the norm.

\subsection{Comparison with numerical simulations}

Several of our observations on ejecta interaction/coalescence are in agreement with observations of H-CMEs near the Sun (Gopalswamy et al., 2001, 2002) and with recent numerical simulations (Odstrcil et al., 2003). The speeding up of the front ejecta and the slowing down of the trailing ejecta was first inferred by Gopalswamy et al. (2001) Odstrcil et al. (2003) employed a 21/2-D MHD numerical code to model shock-cloud and ensuing cloud-cloud interactions. Their magnetic clouds are modeled as cylindricallysymmetric, force-free, constant-alpha (Lundquist) magnetic flux ropes surrounded by a potential field. The clouds are initially at rest. The first cloud (cloud 1) is propelled towards cloud 2 with such a speed that a forward-reverse shock pair is formed. The fast forward shock enters cloud 2. Two sets of cloud 2 parameters are considered: one with a larger, and the other with smaller, characteristic speed. In the simulations, the shock front entering cloud 2 is always distorted in the interaction. In the case where cloud 2 has a smaller characteristic speed, the shock propogates slower in cloud 2 and emerges from the cloud with its lateral wings leading the central portion which has traversed cloud 2. Behind the shock, the density and magnetic field are enhanced. The momentum of the post-shock flow is imparted to cloud 2, accelerating it. The clouds are then pushed into contact. In the assumed configuration, the magnetic field at the leading edge of cloud 1 is oppositely directly to that of the trailing edge of cloud 2. Driven reconnection takes place. The reconnection process proceeds slowly but eventually the two flux tubes coalesce into one and move at a common speed.

Obviously, while the simulation is very idealized, there are many points in common with our observations. There are also some differences to be expected, since the results of the simulations depend strongly on the assumed values of the initial cloud parameters (Odstrcil et al., 2003), such as the orientation of their magnetic fields. For example, in our events no common speed has yet been reached, implying that the merger was not yet complete. Note also that we were unable to show the occurrence of reconnection convincingly, because the measured change in the plasma velocity tangential to the interface $\left(\Delta \mathbf{v}_{t}\right)$ was not correlated with $\Delta \mathbf{B}_{t} / \rho$. This was possibly due to a lack of sufficient magnetic shear across the interface. However, reconnection does, on occasion, occur. Thus, Farrugia et al. (2001) examined a case of a reconnection layer separating ejecta material from other ejecta material in the form of a magnetic cloud. It is thus important to examine other examples where the role of reconnection in the formation of complex ejecta is in evidence.

\subsection{Geoeffects}

The ejecta merger on 31 March 2001 gave rise to a twostep $D_{s t}$ profile. Double-dip storms have been discussed by Kamide et al. (1998). There they are called type 2. According to these authors, the IP cause of type 2 storms are 2 successive intervals of IMF $B_{z}<0$. The huge majority of type 2 storms are found to have the first storm weaker than the second. Only in a small fraction $(8.5 \%$, Kamide et al., 1998 ) is first storm stronger than the second (as we have on 31 March 2001). We suggest that one IP cause of the latter sub-category of geomagnetic storms is ejecta mergers. We can understand this from the foregoing, since the interaction tends to strengthen the field and plasma of the leading ejecta.

Aside from the geoeffects, another reason for studying ejecta-ejecta interactions comes from momentum considerations. In these interactions the ratio of masses expelled by the Sun into IP space in CMEs may be obtained indirectly by evaluating the inverse ratio of the accelerations of interacting pairs of ejecta, assuming elastic collisions.

\subsection{Caveats in the interpretation}

A number of reservations on the interpretations offered here must be borne in mind. In our considerations we were constrained to infer evolutionary changes from two-site, snapshot-like observations. This is a severe constraint in that 
the discrete, two-point measurements did not permit the details of the interaction to be continuously followed. There is also the difference in longitude of $28^{\circ}$ in the 1979 case. Though ejecta longitudinal widths are thought to be larger than this, and those of shocks even more so, it can still pose an interpretational problem. In particular, it is possible that the ejecta seen at one location is absent from the other simply because it does not extend that far in longitude. The works cited in Sect. 2.1 did not assess this issue. We first assumed a merger (like these other authors) and showed that a consistent story can be made. We then searched for Forbush decreases and found them at both locations, further confirming this approach. It might well be, however, that there are elements which we missed precisely because we studied very active periods.

To conclude: We have studied two very active periods which unleashed a number of CMEs into space, some towards the Earth. As such, they are of great interest to the space weather effort. The evolutionary ejecta effects brought to light are large and have important influences on the terrestrial environment. The STEREO mission, with its twinspacecraft capability will be able to continuously monitor ejecta mergers and confirm and extend our inferences directly. Clearly, the interactions complicate space weather forecasting and there is a need of a systematic study to address this issue.

Acknowledgements. We thank R. Schwenn for helpful discussions and for his continued support. Helpful discussions with Len Burlaga are gratefully acknowledged. D. Reames kindly supplied us with the energetic particle data from EPACT/LEMT. We thank the PIs on the four Wind instruments which we employed in this study, and the ACE SCIENCE CENTER at website http: www.srl.caltech.edu/ACE/ASC. We consulted the Solar Geophysical Data Book, published by the US Department of Commerce, National Oceanic and Atmospheric Administration, Environemntal Data and Information Service. The $D_{s t}$ and $K_{p}$ values are courtesy of the Geomagnetic Data Services of Kyoto University at website http://swdcdb.kugi.kyoto-u.ac.jp/wdc/Sec3.html. The top two panels of Fig. 8 are from the Living With A Star - Coordinated Data Analysis Workshop (LWS CDAW) on Solar Energetic Particles: Solar and Geospace Connections, held in Lanham, MD, 22-26 July 2002. The panels were downloaded from URL http://cdaw.gsfc.nasa.gov/LWS/data/event_list.html. This work is supported by NASA grants NAG5-10883, NAG5-11803, NAG513512, NASW-02035, and NSF grants ATM-0309585 and ATM0208414 .

Topical Editor T. Pulkkinen thanks threee referees for their help in evaluating this paper.

\section{References}

Abraham-Shrauner, B.: Determination of magnetohydrodynamic shock normals, J. Geophys. Res., 77, 736-739, 1972.

Berdichevsky, D., Szabo, A., Lepping, R. P., Vinas, A. F., and Mariani, F.: Interplanetary fast shocks and associated drivers observed through the 23rd solar minimum by Wind over its first 2.5 years, J. Geophys. Res., 105, 27 289-27 314, 2000; Erratum in: J. Geophys. Res., 106, 25 133, 2001.
Berdichevsky, D. B., Farrugia, C. J., Thompson, B. J., Lepping, R. P., Reames, D. V., Kaiser, M. L., Steinberg, J. T., Plunkett, S. P., and Michels, D. J.: Halo-coronal mass ejectas near the 23rd solar minimum: lift-off, inner heliosphere, and in situ (1 AU) signatures, Ann. Geophys., 20, 891-916, 2002.

Berdichevsky, D. B., Farrugia, C. J., Lepping, R. P., Galvin, A. B., Schwenn, R., Reames, D. V., Ogilvie, K. W., and Kaiser, M. L.: Solar-heliospheric-magnetospheric observations on March 23April 26, 2001: Similarities to observations in April 1979, Solar Wind 10, edited by: Velli, M., Bruno, R., and Malara, F., 758761, 2003.

Borovsky, J. E., Thomsen, M. F., and Elphic, R. C.: The driving of the plasma sheet by the solar wind, J. Geophys. Res., 103, 17 617-17 639, 1998.

Bougeret, J.-L., Kaiser, M. L., Kellogg, P. J., et al.: The Radio and Plasma Wave Investigation on the Wind spacecraft, Space Sci Rev., 71,, edited by: Russell, C. T., 231-263, 1995.

Burlaga, L. F.: Interplanetary streams and their interaction with earth, Space Sci. Rev., 17, 327-352, 1975.

Burlaga, L. F.: Interplanetary Magnetohydrodynamics, International Series on Astronomy and Astrophysics, Oxford University Press, New York, 1995.

Burlaga, L. F., Sittler, E., Mariani, F., and Schwenn, R.: Magnetic loop behind an interplanetary shock: Voyager, Helios and IMP 8 observations, J. Geophys. Res., 86, 6673-6684, 1981.

Burlaga, L. F., Behannon, K. W., and Klein, L. W.: Compound streams, magnetic clouds, and major geomagnetic storms, J. Geophys. Res., 92, 5725-5734, 1987.

Burlaga, L. F., Lepping, R. P., and Jones, J.: Global configuration of a magnetic cloud, in: Physics of Flux Ropes, Geophys. Monogr. Ser., vol. 58, edited by: Russell, C. T., Priest, E. R., and Lee, L. C.: 373-378, AGU, Washington D.C., 1990.

Burlaga, L. F., Skoug, R. M., Smith, C. W., Webb, D. F., Zurbuchen, T. H., and Reinard, A.: Fast ejecta during the ascending phase of solar cycle 23: ACE observations, 1998-1999, J. Geophys. Res., 106(A10), 20 957-20 977, 2001.

Burlaga, L. F., Plunkett, S. P., and StCyr, P.-C.: Successive CMEs and complex ejecta, J. Geophys. Res., 107, A10, 1266-1677, doi:10.1029/2001JA000255, 2002.

Cane, H. V.: The evolution of interplanetary shocks, J. Geophys. Res., 87, 191-197, 1985.

Cane, H. V., Reames, D. V., and von Rosenwinge, T. T.: The role of interplanetary shocks in the longitude distribution of solar energetic particles, J. Geophys Res., 93, 9555-9567, 1988.

Farrugia, C. J. Vasquez, B., Torbert, R. B., Richardson, I. G., Burlaga, L. F., Biernat, H. K., Mühlebachler, S., Ogilvie, K. W., Lepping, R. P., Berdichevsky, D. B., Kubyshkin, I. G., Lin, R. P., Phan, T.-D., and Scudder, J. D.: A reconnection layer associated with a magnetic cloud, Adv. Space Res., 28, 5, 759-764, 2001.

Fry, C. D., Sun, W., Deehr, C. S., Dryer, M. Smith, Z., Akasofu, S.I., Tokumaru, M. and Kojima, M.: Improvements of the HAF solar wind model for space weather predictions, J. Geophys. Res., 106, 20 985-21 002, 2001.

Gopalswamy, N., Yashiro, S., Kaiser, M. L., Howard, R. A., and Bougeret, J.-L.: Radio signatures of coronal mass ejection interaction: Coronal mass ejection cannibalism?, Astrophy. J., L91L94, 548, 2001.

Gopalsamy, N., Yashiro, S., Kaiser, M. L., Howard, R. A., and Bougeret, J.-L.: Interplanetary radio emission due to interaction between two coronal mass ejections, Geophys. Res. Lett., 29, 1265-1268, doi:10.1029/2001GL013606, 2002. 
Gosling, J. T.: Coronal mass ejections and flux ropes in interplanetary space, in: Physics of Flux Ropes, Geophys. Monogr. Ser., vol. 58, eds. Russell, C. T., Priest, E. R., and Lee, L. C., AGU, Washington D.C., 343-364,1990.

Gosling, J. T., Pizzo, V., and Bame, S. J.: Anomously low proton temperatures in the solar wind following interplanetary shock waves: Evidence for magnetic bottles?, J. Geophys. Res., 78 2001-2009, 1973.

Gosling, J. T., Bame, S. J., McComas, D. J., and Phillips, J. L.,: Coronal mass ejections and large geomagnetic storms, Geophys. Res. Lett., 17, 901-904, 1990.

Gosling, J. T., McComas, D. J., Phillips, J. L., and Bame, S. J.: Geomagnetic activity associated with Earth-passage of interplanetary shock disturbances and coronal mass ejections, J. Geophys. Res., 96, 7831-7839, 1991.

Jordanova, V. K., Farrugia, C. J., Janoo, L., Quinn, J. M., Torbert, R. B., Ogilvie, K. W., Lepping, R. P., Steinberg, J. T., McComas, D. J., and Belian, R. D.: October 1995 magnetic cloud and accompanying storm activity: Ring current evolution, J. Geophys. Res., 103, 79-92, 1998.

Jordanova, V. K., Kistler, L. M., Thomsen, M. F., and Mouikis, C. G.: Effects of plasma sheet variability on the fast initial ring current decay, Geophys. Res. Lett., 30, 1311-1314, doi:10.1029/2002GL016576, 2003.

Kamide, Y., Yokoyama, N., Gonzalez, W., Tsurutani, B. T., Daglis, I. A., Brekke, A., and Masuda, S.: Two-step development of geomagnetic storms, J. Geophys. Res., 103, 6917-6921, 1998.

Klein. L. W. and Burlaga, L. F.: Interplanetary magnetic clouds at 1 AU, J. Geophys. Res., 87, 613-624, 1982.

Lepping, R. P., Acuña, M. H., Burlaga, L. F., et al.: The Wind Magnetic Field Investigation, Space Sci. Rev., 71, 207-229, edited by Russell, C. T., 1995.

Lepping, R. P, Berdichevsky, D. B., Burlaga, L. F., Lazarus, A. J., Kasper, J., Desch, M. D., Wu, C.-C., Reames, D. V., Singer, H. J., Smith, C. W., and Ackerson, K. L.: The Bastille day magnetic clouds and upstream shocks: Near Earth interplanetary observations, Solar Physics, 204, 287-305, 2001.

Lepping, R. P., Berdichevsky, D. B., Szabo, A., Arqueros, C., and Lazarus, A. J.: Profile of an average magnetic cloud at $1 \mathrm{AU}$ for the quiet solar phase: Wind observations, Solar Phys., 212, 425444, 2003.

Lin, R. P., Anerson, K. A., Ashford, S., et al.: A Three-Dimensional Plasma and Energetic Particle Investigation for the Wind spacecraft, Space Sci. Rev., 71, 125-153, 1995.

Lopez, R. E.: Solar cycle invariance in solar wind proton temperature relationships, J. Geophys. Res., 92, 11 187-11 194, 1987.

Lopez, R. E. and Freeman, J. W.: The solar wind proton temperature-velocity relation, J. Geophys. Res., 91, 1701-1705, 1986.

McGuire, R. E., von Rosenwinge, T. T., and McDonald, F. B.: The composition of solar energetic particles, Astrophys. J., 301, 938961, 1986.

Michels, D. J.: Organization of the corona at solar minimum, in: Proc. of the Third Solstip Symposium, 1996, edited by: Feng, X. S., Wei, F. S., and Dryer, M., Int. Acad. Publ., Beijing, China, Plates 1-5, and 34-39, 1998.

Odstrcil, D. and Pizzo, V. J.: Three-dimensional propagation of coronal mass ejections (CMEs) in a structured solar wind flow, 1. CME launced within the streamer belt, J. Geophys. Res., 104, 483-492, 1999a.
Odstrcil, D. and Pizzo, V. J.: Three-dimensional propagation of coronal mass ejections (CMEs) in a structured solar wind flow, 2. CME launced adjacent to the streamer belt, J. Geophys. Res., 104, 493-504, 1999b.

Odstrcil, D. and Pizzo, V. J.: Distortion of the interplanetary magnetic field by three-dimensional propagation of coronal mass ejections in a structured solar wind, J. Geophys. Res., 104, 28 225-28 240, 1999c.

Odstrcil, D., Vandas, M., Pizzo, V. J., and MacNeice, P.: Numerical simulation of interacting magnetic fluc ropes, SOLAR WIND 10, edited by: Velli, M., Bruno, R., and Malara, F., 699-702, 2003.

Ogilvie, K. W., Chornay, D. J., Fitzenreiter, R. J., et al.: SWE, A comprehensive plasma instrument for on the Wind spacecraft, edited by: Russell, C. T., Space Sci. Rev., 71,, 55-77, 1995.

Pizzo, V. J.: Global Modeling of CME propagation in the solar wind, in: Coronal Mass Ejections, edited by: Crooker, N. U., Joselyn, J.-A., and Feynman, J., Geophys. Monogr. Ser., vol. 99, 261-268, AGU, Washington D.C., 1997.

Reames, D. V., Barbier, L. M., and Ng, C. K.: The spatial distribution of particles accelerated by Coronal Mass Ejection-Driven Shocks, Ap. J. 456, 473-486, 1996.

Richardson, I. G. and Cane, H. V.: Regions of abnormally low proton temperature in the solar wind (1965-1991) and their association with ejecta, J. Geophys. Res., 100, 23 397-23 412, 1995.

Richardson, I. G.: Using energetic particles to probe the magnetic topology of ejecta, in: Coronal Mass Ejections, edited by: Crooker, N. U., Jocelyn, J.-A., and Feynmann, J., Geophys. Monogr. Ser., 99, 189-196, AGU, Washington D.C., 1997.

Sonnerup, B. U. Ö and Cahill, L. J.: Magnetopause structure and attitude from Explorer 12 observations, J. Geophys. Res., 72, 171183, 1967.

Sonnerup B. U. Ö., Paschmann, G., Papamastorakis, I., Sckopke, N., Haerendel, G., Bame, S. J., Asbridge, J. R., Gosling, J. T., and Russell, C. T.: Evidence for magnetic field reconnection at the Earth's magnetopause, J. Geophys. Res., 86, 10 049-10 067, 1981.

Steinitz, R. and Menasche, E.: Competing effects determining the Proton temperature gradient in the solar wind, Solar Wind 4, Monogr. MPAE-W-100-81-31, edited by: Rosenbauer, H., Max Planck Institut für Aeronomie, 164-167, 1982.

Sun, W., Dryer, M., Fry, C. D., Deehr, C. S., Smith, Z., Akasofu, S.I., Kartlev, M. D., and Grigorov, K. G.: Real-time forecasting of ICME shock arrrivals at L1 during the "April Fool's Day" epoch: 28 March-21 April, 2001, Ann. Geophys., 20, 937-945, 2002.

Szabo, A.: An improved solution to the "Rankine-Hugoniot" problem, J. Geophys. Res., 99, 14737-14 746, 1994.

Tsurutani, B. T., Gonzalez, W. D., Tang, F., and Lee, Y.-T.: Great magnetic storms, Geophys. Res. Lett., 19, 73-76, 1992.

Vinas, A. F. and Scudder, J. D.: Fast and optimal solution to the "Rankine-Hugoniot Problem", J. Geophys. Res., 91, 39-58, 1986.

Von Rosenwinge, T. T., Barbier, L. M., Karsch, J., et al.: The energetic particles: Acceleration, Composition, and Transport (EPACT) Investigation on the Wind spacecraft, edited by: Russell, C. T., Space Sci. Rev., 71, 155-206, 1995.

Wang, Y. M., Ye, P. Z., and Wang, S.: Multiple magnetic clouds: Several examples during March-April, 2001, J. Geophys. Res., 108, 1370-1381, doi:10.1029/2003JA009850, 2003. 